\title{
The puzzling dredge-up pattern in NGC 1978
}

\author{
M. T. Lederer ${ }^{1}$, T. Lebzelter ${ }^{1}$, S. Cristallo ${ }^{2}$, O. Straniero ${ }^{2}$, K. H. Hinkle ${ }^{3}$, and B. Aringer ${ }^{1,4}$ \\ ${ }^{1}$ University of Vienna, Department of Astronomy, Türkenschanzstraße 17, 1180 Vienna, Austria \\ e-mail: [lederer;lebzelter; aringer]@astro.univie.ac.at \\ 2 INAF, Osservatorio Astronomico di Collurania, 64100 Teramo, Italy \\ e-mail: [cristallo; straniero]@oa-teramo.inaf.it \\ 3 National Optical Astronomy Observatories ${ }^{\star}$, PO Box 26732, Tucson, AZ 85726, USA \\ e-mail: hinkle@noao.edu \\ 4 INAF, Osservatorio Astronomico di Padova, Vicolo dell’Osservatorio 5, 35122 Padova, Italy \\ Received 16 February 2009 / Accepted 2 June 2009
}

\section{ABSTRACT}

\begin{abstract}
Context. Low-mass stars are element factories that efficiently release their products in the final stages of their evolution by means of stellar winds. Since they are large in number, they contribute significantly to the cosmic matter cycle. To assess this contribution quantitatively, it is crucial to obtain a detailed picture of the stellar interior, particularly with regard to nucleosynthesis and mixing mechanisms.

Aims. We seek to benchmark stellar evolutionary models of low-mass stars. In particular, we measure the surface abundance of ${ }^{12} \mathrm{C}$ in thermally pulsing AGB stars with well-known mass and metallicity, which can be used to infer information about the onset and efficiency of the third dredge-up.

Methods. We recorded high-resolution near-infrared spectra of AGB stars in the LMC cluster NGC 1978. The sample comprised both oxygen-rich and carbon-rich stars, and is well-constrained in terms of the stellar mass, metallicity, and age. We derived the $\mathrm{C} / \mathrm{O}$ and ${ }^{12} \mathrm{C} /{ }^{13} \mathrm{C}$ ratio from the target spectra by a comparison to synthetic spectra. Then, we compared the outcomes of stellar evolutionary models with our measurements.

Results. The M stars in NGC 1978 show values of $\mathrm{C} / \mathrm{O}$ and ${ }^{12} \mathrm{C} /{ }^{13} \mathrm{C}$ that can best be explained with moderate extra-mixing on the RGB coupled to a moderate oxygen enhancement in the chemical composition. These oxygen-rich stars do not seem to have undergone third dredge-up episodes (yet). The $\mathrm{C}$ stars show carbon-to-oxygen and carbon isotopic ratios consistent with the occurrence of the third dredge-up. We did not find S stars in this cluster. None of the theoretical schemes that we considered was able to reproduce the observations appropriately. Instead, we discuss some non-standard scenarios to explain the puzzling abundance pattern in NGC 1978.
\end{abstract}

Key words. stars: abundances - stars: AGB and post-AGB - stars: evolution

\section{Introduction}

Modelling the final phases in the evolution of a low-mass star is a demanding task. The stellar interior is a site of rich nucleosynthesis, particularly when the star evolves on the asymptotic giant branch (AGB). The freshly synthesised elements are carried to the outer layers of the star by means of the third dredge-up (TDU, for a review see Busso et al. 1999). The onset and efficiency of this mixing mechanism depends on the mass and the metallicity of the star. Various stellar evolution models developed in the recent past (e.g. Straniero et al. 1997; Herwig 2000; Stancliffe et al. 2004; Straniero et al. 2006; Karakas \& Lattanzio 2007) agree on the theoretical picture of the TDU, but details are still subject to discussions. It is thus important to test the models against observations and to derive constraints to improve our understanding of the involved phenomena and related problems. AGB stars are tracers of intermediate-age stellar populations, which can only be modelled accurately when the evolution of the constituents is known. Moreover, evolved low-mass stars undergo strong mass loss in the late stages of their evolution. They enrich the interstellar medium with the products of nuclear burning and thus, as they are large in number, play a significant role in the cosmic matter cycle.

\footnotetext{
^ Operated by the Association of Universities for Research in Astronomy, under cooperative agreement with the National Science Foundation.
}

The primary indicator of TDU is a carbon surface enhancement. An originally oxygen-rich star can be transformed into a carbon star by an efficient dredge-up of ${ }^{12} \mathrm{C}$ to the surface. The criterion to distinguish between an oxygen-rich and a carbonrich chemistry is the number ratio of carbon to oxygen atoms, $\mathrm{C} / \mathrm{O}$. During the thermally pulsing (TP) AGB phase, while the $\mathrm{C} / \mathrm{O}$ ratio is constantly rising as a consequence of the TDU, there are mechanisms that may counteract the increase in the carbon isotopic ratio ${ }^{12} \mathrm{C} /{ }^{13} \mathrm{C}$. The radiative gap between the convective envelope and the hydrogen burning shell can be bridged by a slow mixing mechanism (cf. Wasserburg et al. 1995; Nollett et al. 2003). In this way, ${ }^{12} \mathrm{C}$ and isotopes of other elements are fed back to nuclear processing. The physical origin of this phenomenon is still not known, which is another argument to establish observational data in order to benchmark theoretical predictions.

Measurements of the carbon abundance and the carbon isotopic ratio have been done for a few bright field AGB stars (Lambert et al. 1986; Harris et al. 1987; Smith \& Lambert 1990). The direct comparison of the values inferred from field star observations to evolutionary models is complicated by the fact that luminosity and mass of those targets can be determined only inaccurately, while both quantities are crucial input parameters for the models. A strategy to circumvent this problem is to observe AGB stars in globular clusters (GC). They provide a rather homogeneous sample with respect to distance, age, mass, and 
metallicity. Admittedly, this simplistic picture is slowly disintegrating. There is evidence that globular clusters often harbour more than one population with abundance variations among the constituents (Mackey \& Broby Nielsen 2007; Piotto et al. 2007; Renzini 2008). The parameters like mass and metallicity are, however, still much better constrained than for field stars. For our purpose, i.e. investigating the abundance variations due to the TDU, the old Milky Way globular clusters are not well suited. The AGB stars of the current generation have an envelope mass too low for TDU to occur, while more massive stars have evolved beyond the AGB phase. The intermediate-age globular clusters in the Large Magellanic Cloud (LMC) serve our needs better. We started with an investigation of the AGB stars in NGC 1846 (Lebzelter et al. 2008, henceforth Paper I) and indeed demonstrated the abundance variation along the AGB due to the TDU.

In this paper, we pursue this idea and study the AGB stars in the LMC globular cluster NGC 1978, deriving C/O and ${ }^{12} \mathrm{C} /{ }^{13} \mathrm{C}$ ratios. We give an overview of previous studies of this cluster and our observations in Sect. 2. Details about the data analysis are given in Sect. 3. In Sect. 3.4 we describe the evolutionary models we use for a comparison to our observational results. The results follow in Sect. 4. We discuss our findings in Sect. 5, giving scenarios on how to interpret the puzzling case of NGC 1978 before we conclude in Sect. 6.

\section{Observations}

\subsection{The target cluster: NGC 1978}

The globular cluster NGC 1978 is a member of the intermediateage $(\tau=1-3 \mathrm{Gyr})$ cluster population (Girardi et al. 1995) in the LMC. This population corresponds to one of the two peaks in the LMC metallicity distribution at $[\mathrm{Fe} / \mathrm{H}]=-0.37$ (with a spread of $\sigma=0.15$ ) reported by Cole et al. (2005). In a recent work, Mucciarelli et al. (2007a) indeed derived a metallicity of $[\mathrm{M} / \mathrm{H}]=-0.37$ that is based on the findings from Ferraro et al. (2006), who claimed $[\mathrm{Fe} / \mathrm{H}]=-0.38 \pm 0.02$ and that $[\alpha / \mathrm{H}]$ is almost solar. In contrast to that, Hill et al. (2000) quoted a grossly deviating value of $[\mathrm{Fe} / \mathrm{H}]=-0.96 \pm 0.20$. They investigated two stars from this cluster (LE8 and LE9, see also Sects. 3 and 4 ) and concluded that $[\mathrm{O} / \mathrm{Fe}]=+0.37 \pm 0.10$. These findings have not been reproduced by other groups. Mucciarelli et al. (2008) rather found a mild depletion in alpha elements on average and ascribe the differences to Hill et al. (2000) to the deviating metallicity. Mucciarelli et al. (2008), who performed detailed abundance studies of red giants with UVES spectra, also did not confirm the results of Alcaíno et al. (1999), who concluded from multicolour CCD photometry that NGC 1978 consists of two sub-populations differing by 0.2 dex in metallicity. According to their work, the north-western half of the cluster is more metal-rich and younger than the south-eastern half (plus differences of the sub-populations in the helium content). This result has to be seen in conjunction with a peculiarity of the cluster, namely its high ellipticity of $\epsilon=0.30^{1}$ which has been found independently by various groups and was most recently confirmed by Mucciarelli et al. (2007a). A merger event that could explain both the elongated shape and the suspected two sub-populations was, however, ruled out for example by Fischer et al. (1992). A tidal interaction with the host galaxy seems to be a more likely explanation for the high ellipticity (van den Bergh 2008, and references therein).

1 The flattening of a globular cluster is defined as $\epsilon \equiv(a-b) / a$, where $a$ is the major axis and $b$ is the minor axis of the cluster.
Mucciarelli et al. (2007a) derived the cluster age, distance modulus, and turn-off mass by fitting isochrones to the cluster colour-magnitude diagram (CMD). The resulting values are $\tau=1.9 \pm 0.1 \mathrm{Gyr},(m-M)_{0}=18.50$ and $M_{\mathrm{TO}}=1.49 M_{\odot}$, respectively, for the best fit found when using isochrones from the Pisa Evolutionary Library (PEL, Castellani et al. 2003). When adopting other isochrones with different overshooting prescriptions, the parameters vary so that one could also derive a somewhat lower turn-off mass $\left(M_{\mathrm{TO}}=1.44 M_{\odot}\right)$, a lower distance modulus $\left((m-M)_{0}=18.38\right)$, and a cluster age from 1.7 to $3.2 \mathrm{Gyr}$.

The various radial velocity measurements quoted in the literature widely agree within the error bars, e.g. Olszewski et al. (1991) and Schommer et al. (1992) derived $\left\langle v_{\mathrm{r}}\right\rangle=+292 \pm$ $1.4 \mathrm{~km} \mathrm{~s}^{-1}$ whereas Ferraro et al. (2006) determined a mean heliocentric velocity of NGC 1978 of $\left\langle v_{\mathrm{r}}\right\rangle=+293.1 \pm 0.9 \mathrm{~km} \mathrm{~s}^{-1}$ and with a velocity dispersion $\sigma=3.1 \mathrm{~km} \mathrm{~s}^{-1}$.

NGC 1978 harbours a number of red giant stars (Lloyd Evans 1980) some of which are known to be carbon stars (Frogel et al. 1990). The easiest explanation for such an occurrence is that these $\mathrm{C}$ stars are AGB stars undergoing the third dredgeup or, alternatively, that they underwent mass accretion from an old AGB companion, which subsequently evolved into a white dwarf. More exotic explanations will be discussed in Sect. 5.2.

\subsection{Spectroscopy}

We recorded high-resolution near-infrared spectra of 12 AGB stars in the LMC globular cluster NGC 1978. Ten stars from our target list were already identified by Lloyd Evans (1980) as red giant stars (and are tagged LE in this work) but largely without information about the spectral type. Frogel et al. (1990) listed a number of AGB stars in NGC 1978, comprising also the stars from LE, and gave information about the spectral type, the cluster membership, and near-infrared photometry data. Based on this information, we selected ten targets that could be observed with the Phoenix spectrograph mounted at the Gemini South Telescope (Hinkle et al. 1998, 2003) with reasonable exposure times. Additionally, we constructed a colour-magnitude diagram from Two Micron All Sky Survey (2MASS, Skrutskie et al. 2006) data and picked two more stars that, judging from their $K$ magnitude and $(J-K)$ colour index, are also located on the AGB of NGC 1978 (stars A and B). Details about the observation targets together with the observing log are given in Table 1. The distribution of all the targets within the cluster is illustrated in Fig. 1.

Our observing programme required observations at two different wavelengths, one in the $H$ band and one in the $K$ band. For this purpose, we utilised the Phoenix order sorting filters H6420 and K4220. However, poor weather conditions in the last of the 4 observing nights prohibited to record $H$-band spectra for the targets LE2 and LE7. For the star LE9, we obtained spectra from queue mode observations in the semester 2008A. The exact wavelength settings are similar to the ones described in Lebzelter et al. (2008), i.e. in the $K$ band our spectra run approximately from 23580 to $23680 \AA$. In the $H$ band (15 530-15 $600 \AA$ ), the spectral region observed was shifted to slightly higher wavelengths to cover a larger part of the $\mathrm{CO} 3-0$ band head at $15581.6 \AA=6417.8 \mathrm{~cm}^{-1}$. The slit width was set to 0.35 (the widest slit) which resulted in a spectral resolution of $R=\lambda / \Delta \lambda=50000$.

The total integration time per target and wavelength setting ranged between 20 (LE3) and 120 (LE9) minutes. For each target we observed at two or three different positions along the slit. 
Table 1. NGC 1978 targets and log of observations (LE stands for Lloyd Evans 1980).

\begin{tabular}{|c|c|c|c|c|c|c|c|c|c|}
\hline \multirow[t]{2}{*}{ ID } & Type & $J$ & $K$ & RA & Dec & $H$ band $\left(\lambda_{\mathrm{c}}=15585 \AA\right)$ & $(S / N)_{\mathrm{H}}$ & $K$ band $\left(\lambda_{c}=23670 \AA\right)$ & $(S / N)_{\mathrm{K}}$ \\
\hline & & \multicolumn{2}{|c|}{ 2MASS } & \multicolumn{2}{|c|}{$\mathrm{J} 2000$} & & & & \\
\hline$\overline{\mathrm{LE} 4}$ & $\bar{M}$ & 12.347 & 11.199 & 052843.72 & -661403.7 & 2006 Dec. 03: $3 \times 1000 \mathrm{~s}$ & 65 & 2006 Dec. 05: $3 \times 1000 \mathrm{~s}$ & 65 \\
\hline LE6 & $\mathrm{C}$ & 12.090 & 10.707 & 052846.27 & -661356.4 & 2006 Dec. 07: $3 \times 800 \mathrm{~s}$ & 70 & 2006 Dec. $06: 3 \times 800 \mathrm{~s}$ & 55 \\
\hline A & M & 12.253 & 11.116 & 052846.23 & -661324.8 & 2006 Dec. $03: 3 \times 1000 \mathrm{~s}$ & 55 & 2006 Dec. $05: 3 \times 1000 \mathrm{~s}$ & 65 \\
\hline LE3 & $\mathrm{C}$ & 11.490 & 9.676 & 052844.49 & -661403.9 & 2006 Dec. 03: $3 \times 400 \mathrm{~s}$ & 105 & 2006 Dec. $05: 3 \times 400 \mathrm{~s}$ & 120 \\
\hline LE10 & M & 12.917 & 11.802 & 052844.44 & -661359.9 & 2006 Dec. $03: 3 \times 1800 \mathrm{~s}$ & 60 & 2006 Dec. $05: 3 \times 1800 \mathrm{~s}$ & 70 \\
\hline LE5 & M & 12.527 & 11.387 & 052843.64 & -661353.0 & 2006 Dec. $03: 3 \times 1200 \mathrm{~s}$ & 50 & 2006 Dec. $05: 3 \times 1200 \mathrm{~s}$ & 60 \\
\hline B & $\mathrm{C}$ & 12.367 & 11.078 & 052843.65 & -661409.6 & 2006 Dec. 07: $3 \times 700 \mathrm{~s}$ & 50 & 2006 Dec. 06: $3 \times 1000 \mathrm{~s}$ & 55 \\
\hline LE1 & $\mathrm{C}$ & 12.282 & 10.467 & 052848.50 & -661459.9 & 2006 Dec. 03: $3 \times 600 \mathrm{~s}$ & 50 & 2006 Dec. 05: $3 \times 600 \mathrm{~s}$ & 115 \\
\hline LE2 & $\mathrm{C}$ & 12.967 & 11.266 & 052848.62 & -661518.7 & - & - & 2006 Dec. $06: 3 \times 1200 \mathrm{~s}$ & 65 \\
\hline LE7 & $\mathrm{C}$ & 12.764 & 11.214 & 052847.85 & -661444.0 & - & - & 2006 Dec. $06: 3 \times 1000 \mathrm{~s}$ & 75 \\
\hline LE8 & M & 13.018 & 11.916 & 052848.47 & -661438.7 & 2006 Dec. 03: $3 \times 2000 \mathrm{~s}$ & 30 & 2006 Dec. $05: 3 \times 2000 \mathrm{~s}$ & 40 \\
\hline LE9 & $\mathrm{M}$ & 13.315 & 12.259 & 052850.73 & -661444.2 & 2008 Mar. 19: $4 \times 1800 \mathrm{~s}$ & 45 & 2006 Dec. $06: 3 \times 2000 \mathrm{~s}$ & 50 \\
\hline
\end{tabular}

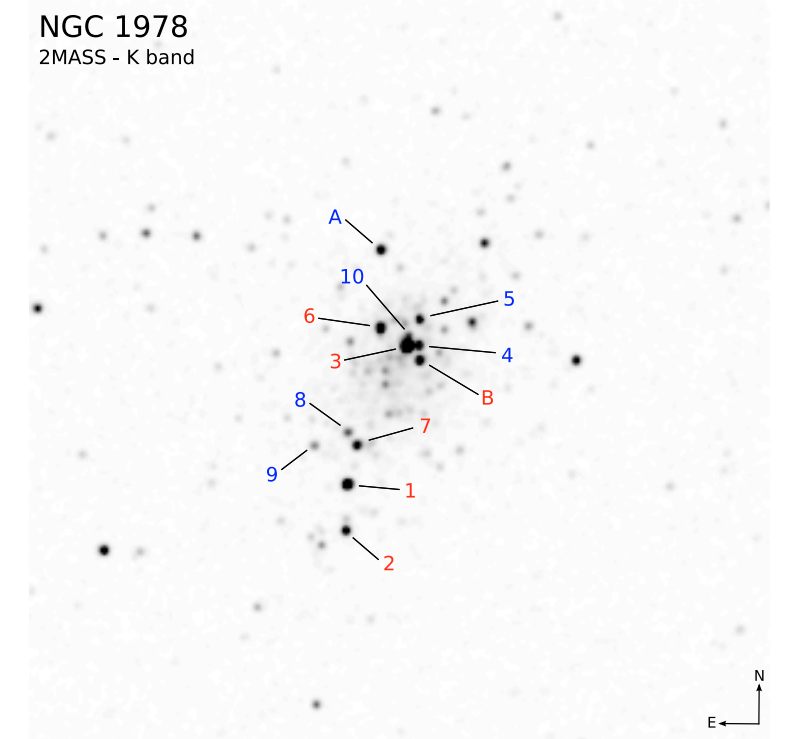

Fig. 1. Distribution of observed targets in the cluster NGC 1978. The picture was acquired from the 2MASS catalogue using Aladin (Bonnarel et al. 2000). Numbers refer to the nomenclature of Lloyd Evans (1980). The additional targets A and B were chosen according to their position in the 2MASS colour-magnitude diagram. The stars A, $4,5,8,9$, and 10 possess an oxygen-rich atmosphere while B, 1, 2, 3,6 , and 7 are carbon stars. The cluster is elongated and its major axis stretches roughly from south-east to north-west. The displayed sky region is $5.0 \times 55^{\prime} .0$ wide. The coordinates of the image centre (close to LE3) read $\alpha=05^{\mathrm{h}} 28^{\mathrm{m}} 45^{\mathrm{s}} .00, \delta=-66^{\circ} 14^{\prime} 14^{\prime \prime} .00$ (J2000).

Each night, we also recorded a spectrum of a hot star without intrinsic lines in the respective wavelength regions in order to correct the spectra of our programme stars for telluric lines. The resulting signal-to-noise was from 50 to above 100 per resolution element ( $\sim 4$ pixels). See Table 1 for details.

\subsection{Data reduction}

The data reduction procedure was carried out as described at length for example in Smith et al. (2002) and in the Phoenix data reduction IRAF tutorial ${ }^{2}$.

\footnotetext{
2 Available at http://www. noao.edu/usgp/phoenix/. IRAF is distributed by the National Optical Astronomy Observatories, which are operated by the Association of Universities for Research in Astronomy, Inc., under cooperative agreement with the National Science Foundation.
}

To correct the AGB star spectra for telluric lines, we acquired the spectrum of an early type star without intrinsic lines. The telluric absorption features were removed using the IRAF task telluric in the $K$ band. The $H$-band spectra are almost free of telluric lines, they were, however, also processed in the same way to remove the fringing. In the $H$ band, we did the wavelength calibration for a K-type radial velocity standard (HD 5457, $v_{\mathrm{r}}=5.1 \mathrm{~km} \mathrm{~s}^{-1}$, Wilson 1953) recorded alongside with the programme stars. Using the Arcturus atlas from Hinkle et al. (1995), we identified several features (OH, Fe, Ti, Si lines) in the spectrum and derived a dispersion solution. The relation was then applied to the remaining spectra. This procedure allowed us to derive radial velocities from the $H$-band spectra. For the $K$-band spectra we did a direct calibration using the CO lines in the spectrum of an M-type target. That solution was then also applied to the carbon-star spectra.

\section{Data analysis}

\subsection{Contents of the observed wavelength ranges}

The wavelength ranges were chosen such that from the $H$ band spectra we could derive the stellar parameters and the $\mathrm{C} / \mathrm{O}$ ratio. Subsequently, we inferred the carbon isotopic ratio ${ }^{12} \mathrm{C} /{ }^{13} \mathrm{C}$ from the $K$ band. In practice, the parameters are tuned iteratively. The region in the $H$ band is widely used in the literature (e.g. Smith et al. 2002; McSaveney et al. 2007; Yong et al. 2008) to derive oxygen abundances from the contained $\mathrm{OH}$ lines. From the relative change of those features in comparison to the band head from the ${ }^{12} \mathrm{C}^{16} \mathrm{O} 3-0$ vibration transition, it is possible to determine the $\mathrm{C} / \mathrm{O}$ ratio in the stellar atmosphere. A weak $\mathrm{CN}$ line and a few metal lines (Fe, $\mathrm{Ti}, \mathrm{Si}$ ) aid in the pinning down of the parameters, especially the effective temperature.

The $K$-band spectra comprise a number of $\mathrm{CO}$ lines from first overtone $(\Delta v=2)$ transitions. Beside features from the main isotopomer ${ }^{12} \mathrm{C}^{16} \mathrm{O}$, we also find some ${ }^{13} \mathrm{C}^{16} \mathrm{O}$ lines in this region. We derived the carbon isotopic ratio by fitting these lines. Apart from the $\mathrm{CO}$ lines, there is also a single HF line blended with a ${ }^{13} \mathrm{CO}$ feature.

In Fig. 2, we give an overview about our observations. For the oxygen-rich stars, the key features in the $H$ band are marked. In the $K$ band, we indicate the position of the ${ }^{13} \mathrm{CO}$ lines. The selection of the wavelength ranges was driven by the oxygen-rich case. From Fig. 2 it is obvious that the regions are not well suited for the analysis of carbon-star spectra. Both $H$ - and $K$-band spectra are crowded with features from the $\mathrm{CN}$ and $\mathrm{C}_{2}$ molecules, occurring in addition to the $\mathrm{CO}$ lines. The polyatomic molecules 


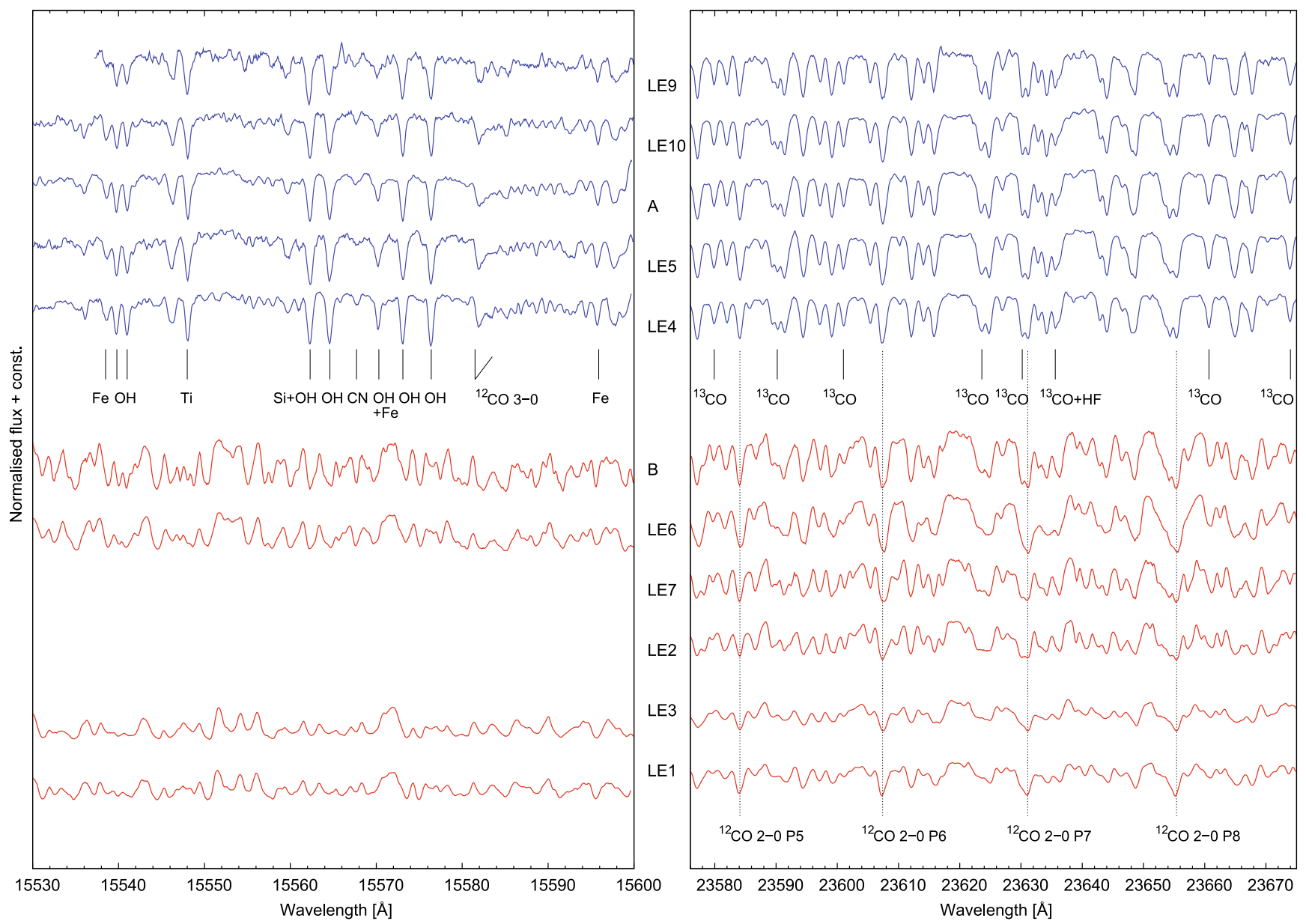

Fig. 2. Overview about observations: $H$-band spectra are shown on the left side, $K$-band spectra on the right. The spectra are ordered by increasing infrared colour $(J-K)_{2 \text { MASS }}$ (from top to bottom), respectively. Some features are identified for the oxygen-rich targets (top), for the carbon-star spectra we only indicate the position of the low-excitation CO lines. Data of the target LE8 are not shown as they are of poor quality and we did not derive abundances from them. $H$-band spectra for the targets LE2 and LE7 could not be taken due to poor weather conditions. See text for details.

$\mathrm{C}_{2} \mathrm{H}_{2}, \mathrm{HCN}$, and also $\mathrm{C}_{3}$ contribute to the opacity by means of many weak lines that form a pseudo-continuum (see Sect. 3.3.2). The major part is due to $\mathrm{C}_{2} \mathrm{H}_{2}$ absorption that increases with decreasing effective temperature. In the $H$ band, the $\mathrm{CO}$ band head is strongly affected by neighbouring features. Generally speaking, there is not a single unblended line in the carbon-rich case. The situation in the $K$ band, however, is not that bad. The ${ }^{12} \mathrm{CO}$ lines are still visible, although blended with $\mathrm{CN}$ features, but there are only a few $\mathrm{C}_{2}$ lines. The pseudo-continuum is essentially all due to $\mathrm{C}_{2} \mathrm{H}_{2}$, contributions from other molecules are negligible.

\subsection{Synthetic spectra}

We compare our observations with synthetic spectra based on model atmospheres that were calculated with the COMARCS code (details will be given in a forthcoming paper by Aringer et al. 2009). COMARCS is a modified version of the MARCS code (originating from Gustafsson et al. 1975; Jørgensen et al. 1992; recently extensively described by Gustafsson et al. 2008). In the model calculations, we deduced the temperature and, accordingly, pressure stratification assuming a spherical configuration in hydrostatic and local thermodynamic equilibrium (LTE). For stars showing only a small variability, LTE is a reasonable approximation. Possible deviations from LTE have a smaller influence on the abundance determination than large deviations from the hydrostatic equilibrium (see for example Johnson 1994 for a discussion of non-LTE effects in cool star atmospheres). In the evaluation of the chemical equilibrium, which is a consequence of LTE, we take all relevant opacity sources into account in both model calculation and spectral synthesis. In the oxygenrich case $\mathrm{H}_{2} \mathrm{O}$, TiO, $\mathrm{CO}$, and $\mathrm{CN}$ are major opacity contributors, while $\mathrm{HCN}, \mathrm{C}_{2} \mathrm{H}_{2}, \mathrm{C}_{2}$ (and others) are important in the carbonrich case. The opacity coefficients utilised by COMARCS are calculated with the COMA code (Aringer 2000; Aringer et al. 2009). The atomic line data were taken from VALD (Kupka et al. 2000), an overview of the molecular line lists used with all the sources documented can be found in Lederer \& Aringer (2009). Model atmospheres and synthetic spectra that were calculated following the method outlined above have been shown to describe the spectra of cool giant stars appropriately (e.g. Loidl et al. 2001; Aringer et al. 2002). We applied the same procedure successfully in Paper I for our analysis of the AGB stars in the LMC cluster NGC 1846.

From the parameters that determine an atmospheric model, we held the mass and the metallicity constant. The respective values are $M=1.5 M_{\odot}$ and $[\mathrm{M} / \mathrm{H}]=-0.4$, and were taken from the literature (see Sect. 1). In the model calculations, the microturbulent velocity was set to $\xi=2.5 \mathrm{~km} \mathrm{~s}^{-1}$, which is in the range that is found for atmospheres of low-mass giants 
(e.g. Aringer et al. 2002; Smith et al. 2002; Gautschy-Loidl et al. 2004). By varying the remaining parameters effective temperature $T_{\text {eff }}[\mathrm{K}]$, logarithm of the surface gravity $\log \left(g\left[\mathrm{~cm} \mathrm{~s}^{-2}\right]\right)$, and carbon-to-oxygen ratio $(\mathrm{C} / \mathrm{O})$, we constructed a grid of model atmospheres and spectra. This was done such that we cover the $T_{\text {eff }}$ and $\log g$ range resulting from colour-temperature relations and bolometric corrections applied to our sample stars. The step size in effective temperature was set to $50 \mathrm{~K}$, whilst the surface gravity was altered in steps of 0.25 on a logarithmic scale, i.e. for $\log g$, ranging from 0.0 to +0.5 . For some carbon stars in our sample we got $\log g<0.0$ from the colour transformations and the adopted mass. The spectral features used in the analysis show only a minor dependence on this parameter thus we fixed $\log g=0.0$ in the analysis of the carbon stars. In this way we also avoid convergence problems for the atmospheric models.

The element composition is scaled solar (Grevesse \& Noels 1993), but we assumed an oxygen over-abundance of +0.2 dex motivated by the results of our work on NGC 1846 (Paper I) and by the paper of Hill et al. (2000). We altered the C/O ratio in steps of 0.05 in the oxygen-rich case and 0.10 in the carbon-rich case. This was done by changing the carbon abundance while leaving the other abundances untouched.

The synthetic spectra cover a wavelength range of $6400-6450 \mathrm{~cm}^{-1}(15504-15625 \AA)$ in the $H$ band and of $4215-4255 \mathrm{~cm}^{-1}(23500-23725 \AA)$ in the $K$ band. The spectra were first calculated with a resolution of $R=\lambda / \Delta \lambda=300000$ and then convolved with a Gaussian ${ }^{3}$ to match the resolution of our observed spectra $(R=50000)$. By applying another convolution with a Gaussian profile we took the macroturbulent velocity into account. To determine the carbon isotopic ratio ${ }^{12} \mathrm{C} /{ }^{13} \mathrm{C}$ (which is about 89.9 in the Sun according to Anders \& Grevesse 1989), we altered this parameter as well. The carbon isotopic ratio has virtually no effect on the model structure and was consequently only considered in the spectral synthesis calculations.

\subsection{Determination of abundance ratios}

We derive the effective temperature $T_{\text {eff }}$, the surface gravity $\log g$, the $\mathrm{C} / \mathrm{O}$ and ${ }^{12} \mathrm{C} /{ }^{13} \mathrm{C}$ ratio of our targets by fitting synthetic spectra to the observations. The initial guesses for $T_{\text {eff }}$ and $\log g$ were obtained using colour-temperature relations and bolometric corrections from the literature. We converted the $K_{2 \text { MASS }}$ and $(J-K)_{2 \text { MASS }}$ values into the Johnson system with the formulae given in Carpenter (2001). For the oxygen-rich targets, we estimated $T_{\text {eff }}$ and $\log g$ from the relations given by Houdashelt et al. (2000). In the case of the carbon stars, we utilised the work of Wood et al. (1983) and Bessell et al. (1983) to obtain bolometric corrections $\left(\mathrm{BC}_{K}\right)$ and effective temperatures, respectively. From the $K$ magnitude together with $\mathrm{BC}_{K}$ and the distance modulus (see Sect. 2) we derived $\log L / L_{\odot}$, and subsequently $\log g$ with the obtained $T_{\text {eff }}$ and by assuming $M=1.5 M_{\odot}$. The reddening of this cluster was taken into account, but it is low $\left(E(B-V)=0.05\right.$ and $A_{K}=0.017$, Grocholski et al. 2007) and has no significant influence on the transformations.

For each target we fit the parameters in a two-step process. The idea is to fit the spectral ranges at once rather than tuning individual abundances (except for carbon) to fit single spectral features. From the $H$ band, we could in this way constrain the stellar parameters $\left(T_{\text {eff }}\right.$ and $\log g$ ) and the $\mathrm{C} / \mathrm{O}$ ratio. Variations in $\log g$

\footnotetext{
3 The Gaussian has the functional form $G(\Delta \lambda, \sigma)=$ $\exp \left\{-(\Delta \lambda / \sigma)^{2} / 2\right\} /(\sigma \sqrt{2 \pi})$, whereby $\sigma_{R} \equiv \lambda /(2 R)$ to reduce the effective resolution to a given $R$, or $\sigma_{v_{\mathrm{t}}} \equiv(\lambda / 2)\left(v_{\mathrm{t}} / c\right)$ to account for the macroturbulent velocity $v_{\mathrm{t}}$.
}

had the smallest effect on the synthetic spectra. A small uncertainty in the stellar mass or radius estimate has thus only a minor influence on the derived abundance ratios. Using the parameters as obtained from the $H$ band, we then calculated $K$-band spectra with a varying ${ }^{12} \mathrm{C} /{ }^{13} \mathrm{C}$ ratio to determine this parameter as well. The carbon isotopic ratio does not influence the appearance of the $H$ band spectrum significantly, as was verified by test calculations.

To match the shape of the spectral features we also had to assume a macroturbulent velocity which reduces the effective resolution of the spectra. This artificial broadening includes the instrumental profile, but it is also used to imitate at least partly the dynamical effects in the stellar atmosphere, which become increasingly important for carbon stars. This is also why the adopted values were generally lower for the oxygen-rich targets: they were fitted by applying a macroturbulent velocity of $v_{\mathrm{t}}=3 \mathrm{~km} \mathrm{~s}^{-1}$. The carbon stars displayed a stronger broadening of the features (cf. Fig. 2). We needed values of $v_{\mathrm{t}}=8 \mathrm{~km} \mathrm{~s}^{-1}$ and even $v_{\mathrm{t}}=10 \mathrm{~km} \mathrm{~s}^{-1}$ (LE6) to fit the spectra (compare also de Laverny et al. 2006 who find comparable values in their study). We consistently applied the same value for the $H$ and the $K$ band.

We tried to objectify the search for the best fit by applying a least-square fitting method. Although we were in this way able to narrow down the possible solutions to a few candidate spectra quickly, the final decision about our best fit was done by visual inspection. The reason is that spectral features with a false strength or position (both due to imperfect line data) in the synthetic spectrum, or spectral regions with a high noise level dominate $\chi^{2}$ and confuse an automatic minimisation algorithm. As the last step in the fitting procedure is done by eye, we assign a formal error to the derived parameters. In the case that the best fit parameters lie in-between our grid values, we quote the arithmetic mean of the candidate models as our fit result. The error bars were estimated from the range of parameters of the model spectra that still gave an acceptable fit.

The formal errors for the derived $\mathrm{C} / \mathrm{O}$ and ${ }^{12} \mathrm{C} /{ }^{13} \mathrm{C}$ ratios given in Table 3 compare well with the uncertainties found by some basic error estimates, as will be shown in the following. Therefore the quintessence of the discussion in Sect. 5 is on a sound footing. We consider typical uncertainties in the stellar parameters and investigate the influence on the derived abundance ratios. The correlations between the stellar parameters and possible systematic errors from the model syntheses have not been taken into account in the error analysis. For the oxygenrich case, we start from a baseline model with $T_{\text {eff }}=3750 \mathrm{~K}$, $v_{\mathrm{t}}=3 \mathrm{~km} \mathrm{~s}^{-1}, \log g=0.25$, and $\mathrm{C} / \mathrm{O}=0.15$. Changes of $\Delta T_{\text {eff }}=100 \mathrm{~K}, \Delta \log g=0.25$, and $\Delta v_{\mathrm{t}}=2 \mathrm{~km} \mathrm{~s}^{-1}$ correspond to changes in $\Delta(\mathrm{C} / \mathrm{O})$ of $0.02,0.03$, and 0.01 , respectively ${ }^{4}$. Summed in quadrature this results in a typical total uncertainty of 0.04 for the $\mathrm{C} / \mathrm{O}$ ratio of our M-type targets. The same exercise for the carbon isotopic ratio results in $\Delta\left({ }^{12} \mathrm{C} /{ }^{13} \mathrm{C}\right)$ of 2 , 2 , and 3 (parameter dependence as above), and thus a total uncertainty of 4 . Concerning the carbon-rich case, we start from a carbon-rich model with parameters $T_{\text {eff }}=3350 \mathrm{~K}, \log g=0.25$, $v_{\mathrm{t}}=8 \mathrm{~km} \mathrm{~s}^{-1}, \mathrm{C} / \mathrm{O}=1.40,{ }^{12} \mathrm{C} /{ }^{13} \mathrm{C}=175$ and vary the parameters as described above. This leads to a total $\Delta(\mathrm{C} / \mathrm{O})=$ $\sqrt{0.1^{2}+0.05^{2}+0.1^{2}}=0.15$. For the carbon isotopic ratio we find $\Delta\left({ }^{12} \mathrm{C} /{ }^{13} \mathrm{C}\right)=\sqrt{15^{2}+15^{2}+30^{2}} \simeq 37$.

\footnotetext{
4 Note that we also include changes of the macroturbulent velocity in the error analysis. The equivalent width of a spectral line is unaffected by $v_{\mathrm{t}}$, but in the spectrum synthesis method uncertainties in this parameter influence the derived abundances.
} 
A considerable part of our discussion will be concerned with the ${ }^{12} \mathrm{C} /{ }^{13} \mathrm{C}$ ratios of our targets. Since the derived isotopic ratios depend on the parameters of the ${ }^{13} \mathrm{CO}$ lines (taken from the list of Goorvitch \& Chackerian 1994, see also Appendix A), we want to assess possible systematic errors in the line strengths. In the case that the predicted line strengths in the list are too large, one would have to increase ${ }^{12} \mathrm{C} /{ }^{13} \mathrm{C}$ (equivalent to a decrease of the ${ }^{13} \mathrm{C}$ abundance) in order to fit a ${ }^{13} \mathrm{CO}$ feature compared with the case where the predicted strengths are correct. The actual ${ }^{12} \mathrm{C} /{ }^{13} \mathrm{C}$ ratio would then be lower than the derived value. For some oxygen-rich stars in our sample we get carbon isotopic ratios that are close to the value of the $\mathrm{CN}$ cycle equilibrium (4-5). A necessary further reduction is thus not very likely. If the theoretical line strengths are too low, the above argument is reversed. One would assume a lower ${ }^{12} \mathrm{C} /{ }^{13} \mathrm{C}$ ratio to fit the ${ }^{13} \mathrm{CO}$ features, which means that one would underestimate the actual isotopic ratio. Since we, of course, use the same line list for the analysis of both $\mathrm{M}$ and $\mathrm{C}$ stars, this would shift up the ${ }^{12} \mathrm{C} /{ }^{13} \mathrm{C}$ for all targets in Figs. 7, 8, and 9. Similar to a $\log g$ uncertainty, the values for the carbon stars would be stronger affected. Hence, underestimated ${ }^{13} \mathrm{CO}$ line strengths would relax the necessity of efficient additional mixing processes on the RGB as discussed in Sect. 5.

In the next section, we discuss $\mathrm{M}$ and $\mathrm{C}$ stars separately. The features contained in the spectra depend on the chemistry regime and some issues cannot be discussed in a general way.

\subsubsection{M-type stars}

In the search for a good fit we took advantage of the way features react to parameter changes. An increasing temperature weakens all features in the $H$ band. The $\mathrm{OH} / \mathrm{Fe}$ blend at approximately $15570 \AA$ is a good temperature indicator. The two neighbouring $\mathrm{OH}$ lines and the $\mathrm{CO}$ band head react less on temperature changes. The remaining lines show only a weak dependence on $T_{\text {eff }}$. The $K$ band is largely insensitive to temperature changes, only the ${ }^{13} \mathrm{CO}$ lines show a weak dependence on temperature which adds to the uncertainty in determining the isotopic ratio. The only feature strongly reacting when altering $T_{\text {eff }}$ in the model calculations is the blend including the HF line, its strength decreases when the temperature is increased.

An increase in $\mathrm{C} / \mathrm{O}$ enhances the strength of the $\mathrm{CO}$ band head and causes stronger $\mathrm{CN}$ lines, while the $\mathrm{OH}$ lines get slightly weaker, especially the feature blended with an Fe line. Lowering $\mathrm{C} / \mathrm{O}$ decreases the strength of the $\mathrm{CO}$ features in the $K$ band.

The $\mathrm{CO}$ band head is also sensitive to changes in $\log g$, while the other features practically are not. Changes in the surface gravity also affect the measured carbon isotopic ratios (see Sect. 3.3). Two of the ${ }^{13} \mathrm{CO}$ lines in the spectra of the oxygenrich stars are unblended $\left({ }^{13} \mathrm{CO} 2-0 \mathrm{R} 18\right.$ and $\left.{ }^{13} \mathrm{CO} 2-0 \mathrm{R} 19\right)$. Blended ${ }^{13} \mathrm{CO}$ features were used to check the isotopic ratio derived from the clean lines.

The continuum is well defined in the oxygen-rich case. We utilised the least-square method in the process of finding the best fit.

\subsubsection{C-type stars}

Several things make the fitting of carbon stars more difficult compared with the case of M-type stars. Foremost, the quality of the line data for the molecules appearing in the carbon-rich case hampers the qualitative analysis profoundly. For $\mathrm{CN}, \mathrm{C}_{3}$, and $\mathrm{C}_{2} \mathrm{H}_{2}$ we used the SCAN database (Jørgensen 1997). The line positions in the computed $\mathrm{CN}$ list are not accurate enough for modelling high-resolution spectra. With the help of measured line positions for $\mathrm{CN}$ that were compiled by Davis et al. (2005), we were able to correct the wavelengths of the strongest lines. While the results were satisfying in the oxygen-rich case, in the carbon-star spectra many additional weak lines show up that could not be corrected. The case of $\mathrm{C}_{2}$ (we used the line data from Querci et al. 1974), producing a wealth of spectral features, is even more problematic: no observed reference line list is available for this molecule, so both line positions and strengths are subject to large uncertainties. We corrected the line list manually by removing strong features that did not appear in any of our observations. Several features were shifted to other wavelengths where it was evident from the observations that the lines are at the wrong position. Unlike for $\mathrm{M}$ stars, the $H$-band spectra of carbon stars are also affected by the carbon isotopic ratio. Lines from ${ }^{13} \mathrm{C}^{12} \mathrm{C}$ or ${ }^{13} \mathrm{C}^{14} \mathrm{~N}$ are important in some blends, however, the quality of the line data for these features could not be assessed for the above described reasons.

The molecules $\mathrm{C}_{2} \mathrm{H}_{2}$ and $\mathrm{C}_{3}$ are incorporated into our calculations via low-resolution opacity sampling data. The absorption thus becomes manifest as a pseudo-continuum in the spectra. A possible occurrence of strong lines or regions with particularly low absorption cannot be reproduced with this approach. The pseudo-continuum level reacts sensitively to temperature changes. In general, an increase in $T_{\text {eff }}$ reduces the feature strength. The relative changes of different line strength can be used to constrain the temperature range. The lower the temperature gets, the higher the contribution of $\mathrm{C}_{2} \mathrm{H}_{2}$ and $\mathrm{C}_{3}$ becomes, whereas $\mathrm{C}_{2} \mathrm{H}_{2}$ dominates the absorption. An increase in the $\mathrm{C} / \mathrm{O}$ ratio has the same effect. The implications for abundance determination of the ill-defined continuum in carbon stars is discussed in detail in Paper I. We want to stress here the consequences for the $\chi^{2}$ method. The absorption in the pseudocontinuum varies with temperature requiring scaling of the observed spectrum before a comparison. Due to this scaling the value of $\chi^{2}$ changes in the same manner, so that model spectra with lower effective temperatures (causing a lower flux level due to an increased pseudo-continuum) always result in a lower $\chi^{2}$, pretending to fit the observations better. This is of course an artificial effect, and thus we cannot rely on the least-square method as objective criterion.

A change in $\log g$ has only small effects on the carbon-star spectra. A higher surface gravity produces a higher pseudocontinuum in the $K$ band. In general $\log g$ only marginally affects the spectral lines. However, in the determination of ${ }^{12} \mathrm{C} /{ }^{13} \mathrm{C}$ the uncertainty in $\log g$ has to be taken into account. In the $K$ band, all ${ }^{13} \mathrm{CO}$ lines are blended with other features. The carbon isotopic ratio is already so high that the strengths of the ${ }^{13} \mathrm{CO}$ lines have become rather insensitive to changes in this parameter. As a consequence, even the small uncertainties in $\log g$ correspond to large changes in the isotopic ratio, which adds to the errors.

Variations of the $\mathrm{C} / \mathrm{O}$ ratio have the strongest impact on the $H$-band spectra when $\mathrm{C} / \mathrm{O}$ is slightly above 1 . The strength of the $\mathrm{CO}$ band head rapidly drops when $\mathrm{C} / \mathrm{O}$ is increased to 1.3, approximately. Then a saturation sets in and the strength of the $\mathrm{CO}$ band head varies slowly with $\mathrm{C} / \mathrm{O}$. This behaviour and strong $\mathrm{CN}$ features sitting in the band head hamper an accurate $\mathrm{C} / \mathrm{O}$ determination. The lines of $\mathrm{C}_{2}$ and $\mathrm{CN}$ increase in strength when $\mathrm{C} / \mathrm{O}$ rises, both in the $H$ and the $K$ band. The $\mathrm{CO}$ lines in the $K$ band decrease in strength for higher values of $\mathrm{C} / \mathrm{O}$. The changes are, however, small and do not allow for a determination of the $\mathrm{C} / \mathrm{O}$ ratio from the $K$-band spectra alone. 
Figure 3 is, as an example, a fit for the star B, which is a carbon star. The star has an effective temperature of about $3350 \mathrm{~K}$ (see also Table 3 for the other fit parameters). The pseudocontinuum contribution is relatively weak, although there are almost no line-free regions in both spectral bands. A number of features is successfully reproduced by our models, the deviations in other regions are most probably due to uncertain line data.

To measure the ${ }^{12} \mathrm{C} /{ }^{13} \mathrm{C}$ ratio in the carbon-star spectra, we utilised the ${ }^{13} \mathrm{CO}$ lines at $23579.9,23590.2$, and $23601.0 \AA$ (the leftmost lines indicated in the right panel of Fig. 2). In the vicinity of the other ${ }^{13} \mathrm{CO}$ lines, there are obviously absorption features missing in our synthetic spectra (see Fig. 3). We cannot rule out that our analysis of a limited number of line blends could introduce a systematic error in the inferred carbon isotopic ratios. Therefore, we emphasise that it would be worthwhile to reassess the isotopic ratios in other wavelength regions, particulary in the light of the discussion in Sect. 5.

We also identified four low-excitation ${ }^{12} \mathrm{CO} 2-0$ lines in the $K$ band (see also Fig. 2). According to Hinkle et al. (1982), lowexcitation lines form in the outer atmospheric layers, opposite to high-excitation lines that show characteristics similar to second overtone transitions $(\Delta v=3)$. In all the carbon-star spectra, these lines could not be fitted with our synthetic spectra. The calculations underestimate the line strength, suggesting that the extended atmospheres of luminous carbon stars are not well reproduced by our hydrostatic models.

\subsection{Stellar evolutionary models}

The stellar evolutionary models presented in this paper have been computed with the FRANEC code (Chieffi et al. 1998). The release we are currently using is optimised to properly compute low-mass models during their AGB phase. Up-to-date input physics, such as low-temperature carbon-enhanced opacities, have been adopted (Cristallo et al. 2007; Lederer \& Aringer 2009). Physical phenomena, such as hydrodynamical instabilities at radiative-convective interfaces and the mass-loss rate, have been properly taken into account (Straniero et al. 2006; Cristallo et al. 2009). The inclusion of an additional mixing mechanism taking place below the convective envelope (usually referred to as extra-mixing) during the RGB and the AGB phase was described in Paper I.

\section{Results}

\subsection{Cluster membership}

We derived the heliocentric radial velocity by cross-correlating the $H$-band spectra with a template spectrum (using the IRAF task fxcor). Data for the star LE9 were taken in the semester 2008A, and no radial velocity standard (K-type star) was recorded, so no information could be deduced for this target. While the quality of our LE8 data is too low to derive abundance ratios, it is still adequate to measure the radial velocity (only with a slightly larger error than for the other targets). The sample shows a relatively homogeneous radial velocity distribution, the spread is quite narrow, so we conclude that all our targets are actually cluster members. The results are summarised in Table 2. The value $v_{\mathrm{r}}=+292.9 \pm 1.3 \mathrm{~km} \mathrm{~s}^{-1}$ (with a velocity dispersion of $\left.\sigma_{v}=2.6 \mathrm{~km} \mathrm{~s}^{-1}\right)$ we find is well in line with earlier determinations (see Sect. 1). As a comparison, Carrera et al. (2008) measured radial velocities in four fields with different distances to the LMC centre. The mean values found in the individual fields
Table 2. Radial velocities of NGC 1978 targets

\begin{tabular}{lccc}
\hline \hline ID & $v_{\mathrm{r}}\left[\mathrm{km} \mathrm{s}^{-1}\right]$ & & \\
\hline A & +295.6 & \pm & 1.0 \\
LE4 & +296.4 & \pm & 0.8 \\
LE5 & +292.9 & \pm & 0.9 \\
LE8 & +290.4 & \pm & 1.3 \\
LE10 & +292.5 & \pm & 0.7 \\
& & & \\
B & +290.6 & \pm & 1.3 \\
LE1 & +297.5 & \pm & 1.6 \\
LE3 & +291.5 & \pm & 1.9 \\
LE6 & +290.2 & \pm & 1.7 \\
\hline mean & $+\mathbf{2 9 2 . 9}$ & \pm & $\mathbf{1 . 3}$ \\
velocity dispersion $(\sigma)$ & & & 2.6 \\
\hline
\end{tabular}

range from +278 to $+293 \mathrm{~km} \mathrm{~s}^{-1}$. The velocity dispersion in the metallicity bin with $[\mathrm{Fe} / \mathrm{H}] \geq-0.5$ is $\sigma_{v}=20.5 \mathrm{~km} \mathrm{~s}^{-1}$.

The errors for the carbon stars are systematically larger than for the oxygen-rich stars. This can easily be understood by looking at Fig. 2. The features in the C-rich case are usually broader and consequently the peak in the cross-correlation function (and thus the FWHM) is broader, too.

\subsection{Stellar parameters and abundance ratios}

We summarise our fit results in Table 3. In the first column, we list the star identifier (compare Fig. 1), followed by the $K$ magnitude and the colour index $(J-K)$ taken from the 2MASS database. In the next two columns, we quote the effective temperature and luminosity (rounded to $100 L_{\odot}$ ) resulting from the respective colour calibrations and bolometric corrections (see Sect. 3.3). The parameters resulting from our fitting procedure $\left(T_{\text {eff }}, \log g, v_{\mathrm{t}}, \mathrm{C} / \mathrm{O},{ }^{12} \mathrm{C} /{ }^{13} \mathrm{C}\right)$ are listed in the subsequent columns. We note that none of our target stars is a large amplitude variable, thus the influence of variability on the stellar parameters can be safely ignored. A detailed discussion of the small amplitude variability $(1.5 \mathrm{mag}$ in $R$ ) will be given elsewhere (Wood \& Lebzelter, in preparation).

The derived effective temperatures are systematically higher than the $T_{\text {eff }}$ values inferred from the infrared colour transformations (compare the resulting $T_{\text {eff,c }}$ to $T_{\text {eff,f }}$ deduced from the fit in Table 3). Better agreement between the two temperature scales is found when we adopt a scaled solar oxygen abundance rather than an over-abundance of $0.2 \mathrm{dex}$, which was our standard choice in the analysis (we refer to Sects. 1 and 3.2 for details). However, this is not too surprising since the colourtemperature relations from Houdashelt et al. (2000) were derived from synthetic spectra based on scaled solar abundances. We want to mention that the results from Smith et al. (2002, their Fig. 8) imply $[\mathrm{O} / \mathrm{Fe}] \leq 0.0$ for the LMC, contrary to our assumption. Also, Mucciarelli et al. (2008) found that the other alpha element abundances are roughly scaled solar $([\alpha / \mathrm{Fe}] \simeq 0.0)$ in NGC 1978. However, in another recent work Goudfrooij et al. (2009) derived $[\alpha / \mathrm{Fe}]=0.2$ for the LMC cluster NGC 1846. Apart from the influence on the temperature scale, a higher oxygen abundance would shift the derived $\mathrm{C} / \mathrm{O}$ ratios only to slightly higher values (Fig. 4). The derived carbon isotopic ratios are marginally affected by an oxygen over-abundance.

We found that for the five oxygen-rich stars within our sample the $\mathrm{C} / \mathrm{O}$ ratio is varying little, the values range from 0.13 to 0.18 with a typical uncertainty of \pm 0.05 . The isotopic ratios vary in the range between 9 and 16 with an uncertainty ranging 


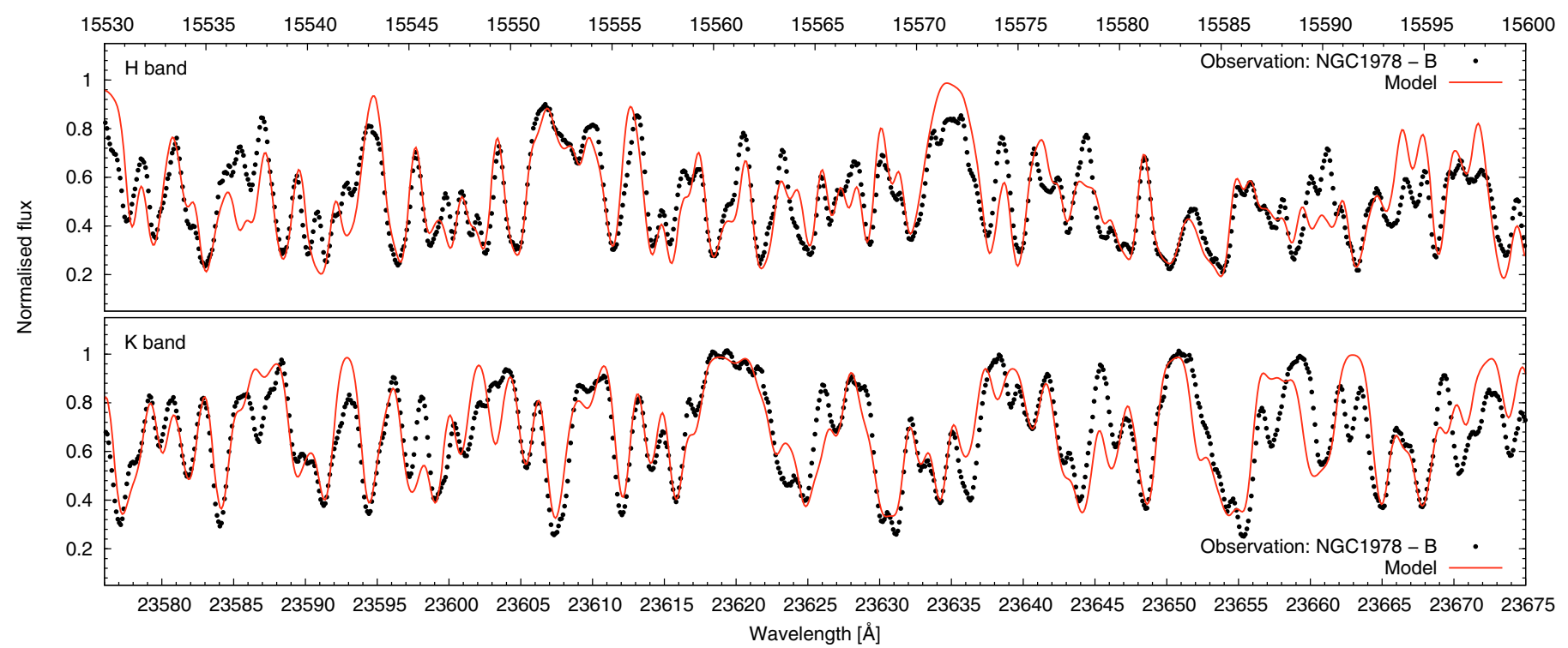

Fig. 3. Observation and fit of the carbon star B in NGC 1978. The fit parameters are given in Table 3. The relatively high effective temperature and low $\mathrm{C} / \mathrm{O}$ ratio compared with the other carbon stars in our sample allowed a reasonable fit. Deviations can to a high fraction be ascribed to uncertain line data. The strong low-excitation CO lines in the $K$ band are not reproduced by the models. See text for details.

Table 3. Data and fit results for oxygen-rich (first group) and carbon-rich (second group) targets in NGC 1978.

\begin{tabular}{|c|c|c|c|c|c|c|c|c|c|}
\hline$\overline{\mathrm{ID}}$ & $\begin{array}{c}K \\
2 \mathrm{M}\end{array}$ & $\begin{array}{l}J-K \\
\mathrm{SS}\end{array}$ & $\begin{array}{c}T_{\text {eff,c }} \\
{[\mathrm{K}]}\end{array}$ & $\overline{\bar{L} L / L_{\odot}}$ & $\begin{array}{c}T_{\text {eff,f }} \\
{[\mathrm{K}]}\end{array}$ & $\log g$ & $\begin{array}{r}v_{\mathrm{t}} \\
{\left[\mathrm{km} \mathrm{s}^{-1}\right]}\end{array}$ & $\overline{\mathrm{C} / \mathrm{O}}$ & ${ }^{12} \mathrm{C} /{ }^{13} \mathrm{C}$ \\
\hline $\mathrm{A}$ & 11.116 & 1.137 & 3600 & 5300 & 3825 & 0.50 & 3 & $0.23 \pm 0.05$ & $16 \pm 3$ \\
\hline LE4 & 11.199 & 1.148 & 3600 & 5000 & 3725 & 0.38 & 3 & $0.18 \pm 0.03$ & $13 \pm 4$ \\
\hline LE5 & 11.387 & 1.140 & 3600 & 4200 & 3775 & 0.25 & 3 & $0.18 \pm 0.05$ & $6 \pm 2$ \\
\hline LE9 & 12.259 & 1.056 & 3800 & 2000 & 3900 & 0.38 & 3 & $0.13 \pm 0.03$ & $12 \pm 2$ \\
\hline LE10 & 11.802 & 1.115 & 3700 & 3300 & 3900 & 0.38 & 3 & $0.18 \pm 0.05$ & $9 \pm 3$ \\
\hline B & 11.078 & 1.289 & 3176 & 4400 & 3350 & 0.00 & 8 & $1.35 \pm 0.10$ & $175 \pm 25$ \\
\hline LE1 & 10.467 & 1.815 & 2554 & 6200 & 2600 & 0.00 & 8 & $>1.50$ & $>200$ \\
\hline LE3 & 9.676 & 1.814 & 2556 & 12900 & 2600 & 0.00 & 8 & $>1.50$ & $>200$ \\
\hline LE6 & 10.707 & 1.383 & 3043 & 5800 & 3100 & 0.00 & 10 & $1.30 \pm 0.10$ & $150 \pm 50$ \\
\hline
\end{tabular}

up to \pm 4 . Considering the error bars, all $\mathrm{M}$ stars occupy more or less the same region in Fig. 5, where we display the measured $\mathrm{C} / \mathrm{O}$ and ${ }^{12} \mathrm{C} /{ }^{13} \mathrm{C}$ of our sample stars. No target is offset from the spot marking the abundance ratios expected after the evolution on the first giant branch $\left(\mathrm{C} / \mathrm{O} \simeq 0.2,{ }^{12} \mathrm{C} /{ }^{13} \mathrm{C} \simeq 10\right)$. In other words, we do not find conclusive signs of third dredge-up among the oxygen-rich stars in the cluster. This is also consistent with the results from Lloyd Evans (1983) who did not find S-type stars in NGC 1978. The total number of our targets is small, so general statements based on our results are rather weak due to the poor statistics. However, the fact that we find oxygenrich stars, in which the TDU does not seem to be active, does not exclude that this phenomenon is at work in other stars of the cluster.

In fact, we also identify a sub-sample of carbon-rich stars belonging to NGC 1978. Satisfactory fits could only be achieved for the two hottest carbon stars (B and LE6) in our sample. We also derived $\mathrm{C} / \mathrm{O}$ and ${ }^{12} \mathrm{C} /{ }^{13} \mathrm{C}$. The error bars are larger than for the $\mathrm{M}$ stars, the reasons for that are outlined in the previous sections. For the two cool objects LE1 and LE3, we could only derive lower limits for $\mathrm{C} / \mathrm{O}$ and ${ }^{12} \mathrm{C} /{ }^{13} \mathrm{C}$. For an increasing carbon content, the features saturate, thus we cannot give a reliable upper limit for $\mathrm{C} / \mathrm{O}$. The $\mathrm{C} / \mathrm{O}$ ratios that we found are in line with the results obtained by other groups.
Matsuura et al. (2005) adopted C/O $\geq 1.4$ to explain molecular features in low-resolution spectra of LMC carbon stars. Investigations of planetary nebulae in the LMC exhibit a range of $\mathrm{C} / \mathrm{O}$ ratios from slightly above 1 up to even 5 (e.g. Stanghellini et al. 2005). We are not aware of measurements of the carbon isotopic ratio directly from carbon-rich AGB stars in the LMC other than the ones presented here and in Paper I. Reyniers et al. (2007) analysed the post-AGB object MACHO 47.2496.8 $([\mathrm{Fe} / \mathrm{H}]=-1.4)$ in the $\mathrm{LMC}$ and derived $\mathrm{C} / \mathrm{O}>2$ and ${ }^{12} \mathrm{C} /{ }^{13} \mathrm{C} \sim 200$. A peculiar combination of $\mathrm{C} / \mathrm{O}$ and ${ }^{12} \mathrm{C} /{ }^{13} \mathrm{C}$ values even more extreme than those of our carbon-rich targets was presented by de Laverny et al. (2006). They found $\mathrm{C} / \mathrm{O} \leq 1.2$ and ${ }^{12} \mathrm{C} /{ }^{13} \mathrm{C}>300$ for BMB-B 30 (with $[\mathrm{M} / \mathrm{H}]=-1.0$ ) in the SMC.

Qualitative estimates based on the $K$-band spectra of the targets LE2 and LE7 suggest that those have an effective temperature between the groups LE1-LE3 and B-LE6 (see Fig. 2). Except for the target B, the difference between the derived effective temperature and the $T_{\text {eff }}$ value obtained from the colour transformation is not quite as high as in the oxygen-rich case (about $50 \mathrm{~K})$. We repeated the analysis for the star B with a scaled solar oxygen abundance (as for LE5, Fig. 4) and also found a lower $T_{\text {eff }}$, whereas the $\mathrm{C} / \mathrm{O}$ ratio remained basically unchanged. Almost all spectral features in the carbon-rich case 


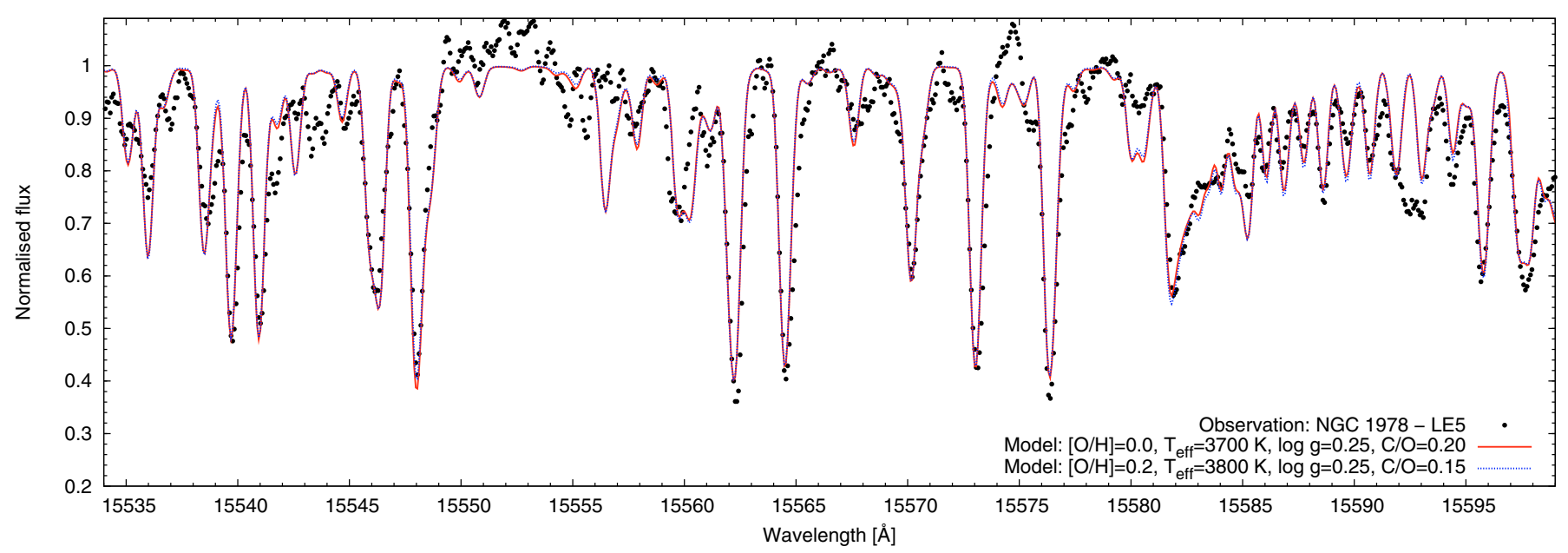

Fig. 4. Fit of the $H$ band spectrum of the target LE5 adopting different oxygen abundances. A value of $[\mathrm{O} / \mathrm{Fe}]=0.2$ (which we used in our analysis) leads to a higher temperature and a lower $\mathrm{C} / \mathrm{O}$ ratio compared with a scaled solar oxygen abundance $([\mathrm{O} / \mathrm{Fe}]=0.0)$. The two model spectra both give reasonable fits to the observations, thus a decision about the actual oxygen abundance cannot be made from our data. However, lower effective temperatures (and thus the "no oxygen over-abundance" scenario) agree better with the values derived from colour-temperature relations.

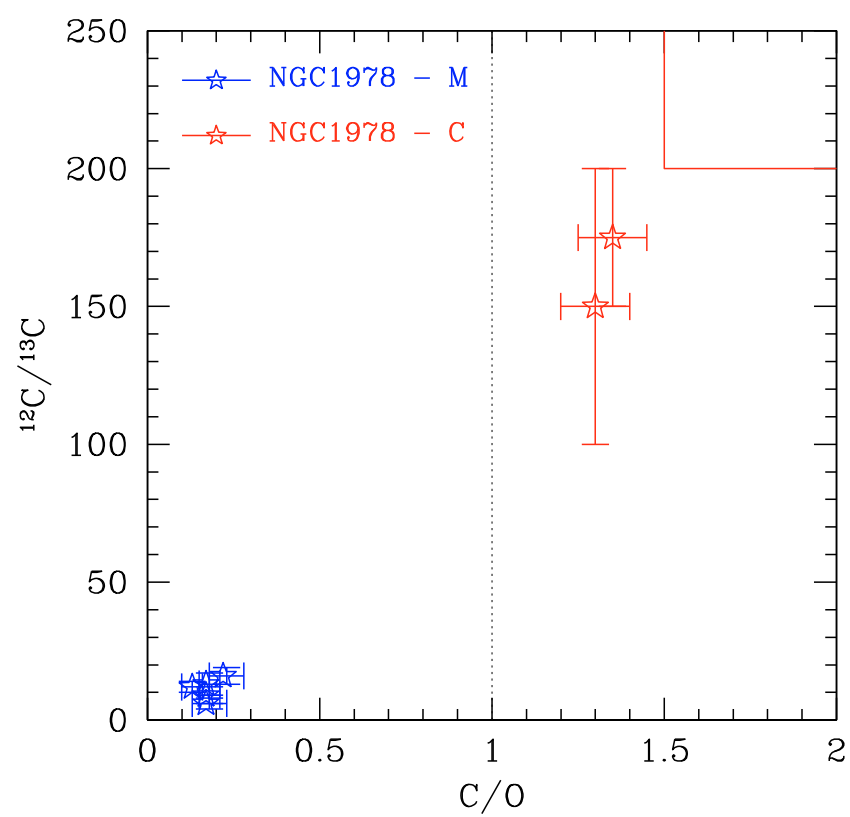

Fig. 5. Measured abundances ratios $\mathrm{C} / \mathrm{O}$ and ${ }^{12} \mathrm{C} /{ }^{13} \mathrm{C}$ for the targets in NGC 1978. Oxygen-rich targets appear to the left of the dotted line at $\mathrm{C} / \mathrm{O}=1$, carbon-rich targets are on the right. The box in the upper right corner marks the lower limits for the targets LE1 and LE3. There are no obvious signs of TDU among the M stars (see left panel of Fig. 7 for a zoom to the M-star data points), S stars are completely lacking in our sample of cluster stars. Additionally, from the carbon-star data we cannot detect a saturation level for the carbon isotopic ratio which would hint at the occurrence of extra-mixing.

react sensitively to temperature changes, therefore $T_{\text {eff }}$ can be better defined than the $\mathrm{C} / \mathrm{O}$ ratio. A remarkable result is that opposite to our findings for NGC 1846 (Paper I) - we do not find a saturation level for the carbon isotopic ratio. This indicates that extra-mixing is not at work in NGC 1978.

In Fig. 6 we show a CMD of NGC 1978 (see Sect. 5 for a discussion of the photometric errors). We do not plot

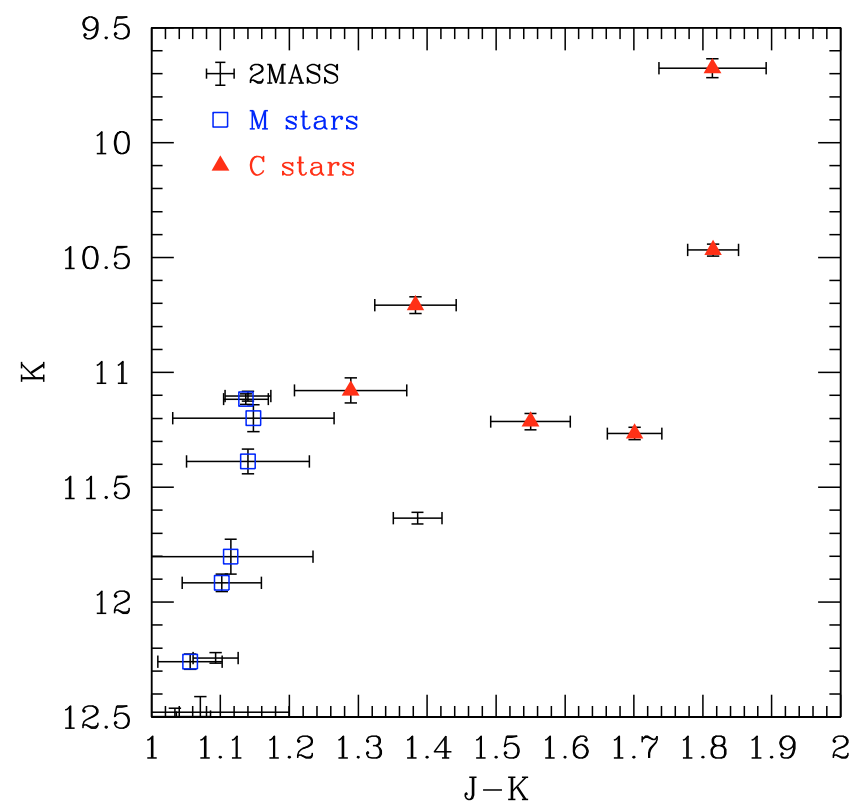

Fig. 6. Colour-magnitude diagram based on 2MASS data for NGC 1978 (complete within a radius of 4'0 with respect to the cluster centre). Empty squares refer to oxygen-rich objects in our sample, filled triangles depict carbon stars. Compare also Table 3. Details are discussed in the text.

the bolometric magnitudes on the ordinate because of the inconsistent bolometric corrections applied to the targets. The CMD contains all targets that lie within a radius of 4.0 with respect to the cluster centre (cf. Fig. 1). Data points with superimposed symbols refer to stars contained in our sample. The oxygen-rich stars (empty squares) seem to form a sequence with a $\mathrm{C} / \mathrm{O}$ ratio increasing with luminosity which might hint at a mild dredge-up. However, this could be misleading, as we have to take the error bars quoted in Table 3 into account. Moreover, the isotopic ratios do not appear in an ordered sequence. The carbon stars (red triangles) are brighter in $K$ and have significantly redder colours. 

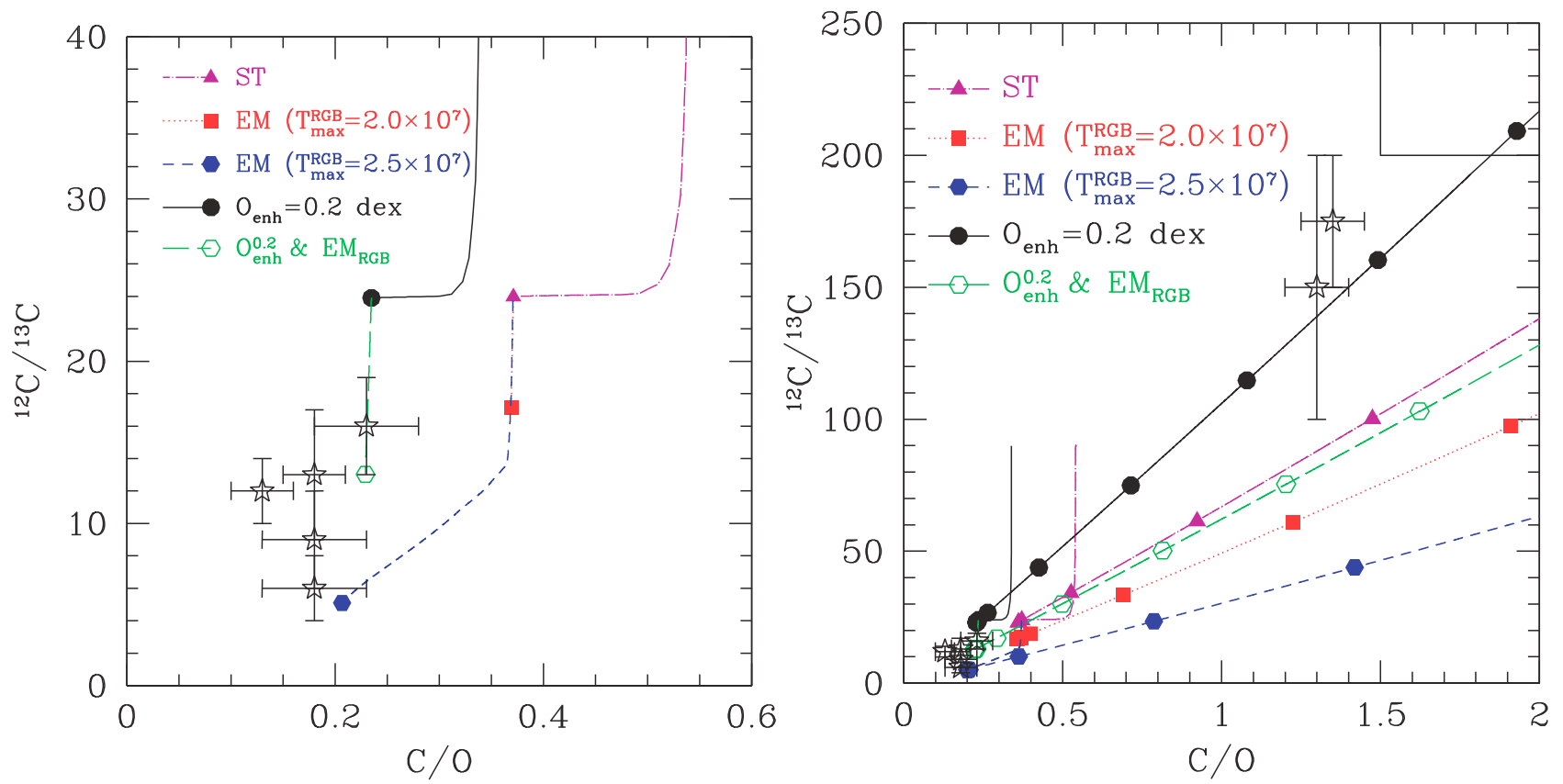

Fig. 7. Comparison between observational data and theoretical models. ${ }^{12} \mathrm{C} /{ }^{13} \mathrm{C}$ isotopic ratios vs. $\mathrm{C} / \mathrm{O}$ ratios are reported. In the left panel we report the range of O-rich stars only, while in the right panel the whole sample has been considered. The star symbols with error bars indicate our observations. The red dotted, the blue dashed, and the green long-dashed curves in the left panel are partly covered by other tracks in the plot. The symbols in the left panel mark the end point of the tracks after the RGB phase. In the right panel, the symbols indicate the values attained during the interpulse phases, which are on the order of $10^{3}$ times longer than the TDU episodes. See text for details.

For the stars LE1 and LE3, having the highest $(J-K)$ values, the indicated numbers refer to lower limits for the abundance ratios. The two carbon stars with the lowest $K$ magnitude are LE2 and LE7 which were excluded from our analysis. Both have luminosities comparable to the brightest oxygen-rich stars. A possible explanation is that these stars are in a post-flash dip phase (Iben \& Renzini 1983), displacing them about 1 magnitude down from the average luminosity in this evolutionary stage in the CMD (see also the discussion in Paper I).

\section{Discussion}

We seek to explain the following observed features of NGC 1978:

1. the cluster harbours carbon stars;

2. the M stars do not show conclusive signs of third dredge-up;

3 . there is no saturation of the carbon isotopic ratio.

Except for the first point, this is more or less the opposite of what we found for the cluster NGC 1846 (Paper I), the composition of which is similar to the one of NGC 1978, even if the GC is slightly younger and, as a consequence, harbours more massive AGB stars (with a mass of about $1.8 M_{\odot}$ ). The $\mathrm{M}$ stars in NGC 1846 span a range in $\mathrm{C} / \mathrm{O}$ from 0.2 to 0.65 , and ${ }^{12} \mathrm{C} /{ }^{13} \mathrm{C}$ - initially rising together with $\mathrm{C} / \mathrm{O}$ - does not exceed 60 to 70 , even in the carbon stars.

The first point in the list is a clear indication of ongoing TDU in the cluster. The second point may at first seem to contrast this statement, while low number statistics could resolve this apparent contradiction: the lifetime of a thermally pulsing AGB star is short, so in a sample of a few stars we do not necessarily have to find a star with an enhanced carbon abundance. This possibly explains the large gap between the $\mathrm{M}$ and the $\mathrm{C}$ stars in terms of $\mathrm{C} / \mathrm{O}$ (see Fig. 5). An alternative scenario where the TDU is so efficient that the star becomes carbon-rich after a single dredgeup episode occurs only at much lower metallicity (e.g. Herwig et al. 2000).

Let us put aside the $\mathrm{C} / \mathrm{O}$ error bars for a moment and speculate about the group of M stars. The 2MASS data come with uncertainties in $K$ of lower than 0.05 , except for LE10 where the error is $0.076^{5}$ In any case, the luminosity sequence that the stars form is conserved. The star LE9 is the faintest at $K$, with $m_{K}=12.3$, in the sample and also the star with the lowest C/O ratio. Cioni et al. (2000) identify the RGB tip in the LMC at $K_{0}=12.1 \mathrm{mag}$. According to this value, LE9 could as well be an RGB star or located at the early-AGB. The targets LE5 and LE10 are possibly at the very beginning of the thermally pulsing asymptotic giant branch (TP-AGB) star phase. The case of LE4 and, in particular, A is more difficult. Comparing these stars with those of similar luminosity in the sample of NGC 1846, we note that the latter are classified as $\mathrm{S}$ stars and present $\mathrm{C} / \mathrm{O}$ ratios of 0.4 or even higher. The $\mathrm{C} / \mathrm{O}$ ratio we find for the star A (0.23) is slightly higher than the average of the other stars, perhaps compatible with just one (the first) TDU episode. The bolometric magnitude of A also roughly corresponds to the limit we found for the onset of the TDU in NGC $1846\left(m_{\mathrm{bol}}=14.1 \mathrm{mag}\right)$.

\subsection{Stellar evolutionary models}

In this section we present theoretical tracks computed with the FRANEC code, and we compare them with observations. In the left panel of Fig. 7, we report the evolution of the ${ }^{12} \mathrm{C} /{ }^{13} \mathrm{C}$ versus $\mathrm{C} / \mathrm{O}$ curves from the pre-main sequence up to the early-AGB phase in the region where the M stars of NGC 1978 lie. In practice, the end point of each curve (marked by a symbol in the left

5 The errors for $(J-K)$ are higher, ranging up to and being even greater than 0.1. This could also affect the differences between the effective temperature scales discussed in Sect. 4. 
panel of Fig. 7) represents the value attained at the tip of the RGB phase, which is conserved up to the onset of the first thermal pulse. In the right panel, instead, we extend the axes to also include the C-rich stars. We plot the whole AGB evolutionary tracks and mark the values attained after each TDU episode.

We firstly concentrate on the O-rich stars of our sample. We carry on our analysis under the assumption that these stars have not experienced TDU, because they still are at the beginning of the TP-AGB phase or because they arrived on the AGB with a too small envelope mass. In this case, their initial surface composition has been modified by the occurrence of the First Dredge-up (FDU) only and, eventually, by an additional slow mixing operating below the convective envelope during the RGB phase (the so called extra-mixing, see e.g. Nollett et al. 2003; Charbonnel \& Zahn 2007; Denissenkov \& Pinsonneault 2008). Our reference model (ST, dash-dotted magenta curve) has an initial mass $M=1.5 M_{\odot}$ with $Z=0.006$, corresponding to $[\mathrm{Fe} / \mathrm{H}]=-0.36$. We assume an initial solar-scaled composition, which implies $\mathrm{C} / \mathrm{O}=0.5$ and ${ }^{12} \mathrm{C} /{ }^{13} \mathrm{C}=90$. After the FDU, the surface values attain $\mathrm{C} / \mathrm{O}=0.36$ and ${ }^{12} \mathrm{C} /{ }^{13} \mathrm{C}=24$. This is due to the fact that the convective envelope penetrates into regions where partial hydrogen-burning occurred before. No extramixing has been included in the ST case. The values so obtained clearly disagree with the $\mathrm{M}$ stars observations, which show an average $\mathrm{C} / \mathrm{O}=0.18$ and an average ${ }^{12} \mathrm{C} /{ }^{13} \mathrm{C}=11$. Thus, we explored the possibility of an occurrence of extra-mixing on the RGB.

This hypothesis is supported by the bulk of observations of RGB stars in the galactic field, as well as in open and globular clusters (see e.g. Gratton et al. 2000). These observations show that this additional mixing occurs in low-mass stars $\left(M<2 M_{\odot}\right)$ during the first ascent along the Red Giant Branch. Moreover, Eggleton et al. (2008) identified a mixing mechanism driven by a molecular weight inversion $(\delta \mu$-mixing $)$ in three-dimensional stellar models that must operate in all low-mass stars while they are on the RGB. The operation of this RGB extra-mixing is also required to explain the relatively low ${ }^{12} \mathrm{C} /{ }^{13} \mathrm{C}$ ratios in the M stars of NGC 1846 (Paper I).

As in Paper I, we include this additional mixing right after the RGB luminosity bump. The extension of the zone in which this additional mixing takes place is fixed by prescribing the maximum temperature the material is exposed to $\left(T_{\mathrm{RGB}}^{\max }\right)$. The circulation rate is tuned by setting the mixing velocity to a value that is a small fraction (cf. Paper I) of the typical convective velocities in the envelope of an RGB star. As discussed in Paper I, the carbon isotopic ratio largely depends on $T_{\mathrm{RGB}}^{\mathrm{max}}$. In Fig. 7 we report two models, characterised by $T_{\mathrm{RGB}}^{\max }=2.0 \times 10^{7} \mathrm{~K}$ (red dotted curve) and $T_{\mathrm{RGB}}^{\max }=2.5 \times 10^{7} \mathrm{~K}$ (blue short-dashed curve). In the first case, the final ${ }^{12} \mathrm{C} /{ }^{13} \mathrm{C}$ ratio decreases, reaching a value in good agreement with those observed in the $\mathrm{M}$ stars of NGC 1978. Notwithstanding, the C/O ratio remains unaltered and higher than the observed one. An increase of $T_{\mathrm{RGB}}^{\max }$ up to $2.5 \times 10^{7} \mathrm{~K}$ leads to a lower $\mathrm{C} / \mathrm{O}$ ratio of about 0.21 , which is in better agreement with the observations. However, the corresponding ${ }^{12} \mathrm{C} /{ }^{13} \mathrm{C}$ ratio is 5 , which is definitely lower than the average value (11). Note that only LE5 shows such a low value $\left({ }^{12} \mathrm{C} /{ }^{13} \mathrm{C}=6\right)$.

Then, we explored the possible effect of an oxygen enhancement (see the discussion in Sect. 2.1). The black solid line in Fig. 7 represents a model similar to the ST case, but with $[\mathrm{O} / \mathrm{Fe}]=0.2$. As for the ST case, the effects of the FDU are clearly recognisable. An additional model, as obtained by including an RGB extra-mixing $\left(T_{\mathrm{RGB}}^{\max }=2.1 \times 10^{7} \mathrm{~K}\right)$ is also reported (green line). The final surface composition of this model $\left(\mathrm{C} / \mathrm{O}=0.23\right.$ and $\left.{ }^{12} \mathrm{C} /{ }^{13} \mathrm{C}=13\right)$ is close to the average values of the observed sample $\left(\mathrm{C} / \mathrm{O}=0.18\right.$ and $\left.{ }^{12} \mathrm{C} /{ }^{13} \mathrm{C}=11\right)$. Thus, a first conclusion is that moderate RGB extra-mixing $\left(2.1 \times 10^{7}<T_{\mathrm{RGB}}^{\max }<2.3 \times 10^{7} \mathrm{~K}\right)$ coupled to moderate oxygen enhancement appears to nicely reproduce the observed average composition of the M stars in NGC 1978. The same conclusion has been reached in the case of NGC 1846 (Paper I).

What are the implications of this scenario when applied to the whole observational sample (O-rich and C-rich stars)? We carry on our analysis assuming that the carbon stars are intrinsic, i.e. that their surface composition, in particular the high $\mathrm{C} / \mathrm{O}$ and carbon isotopic ratios, is the result of nucleosynthesis and mixing processes occurring during the thermally pulsing AGB evolution.

In the right panel of Fig. 7 we report the same models as shown in the left panel. None of the theoretical tracks can simultaneously reproduce the abundance ratios of the $\mathrm{M}$ and $\mathrm{C}$ stars. The high values of the $\mathrm{C}$-star carbon isotopic ratio can only be reproduced by the oxygen-enhanced model with no RGB extra-mixing. All the other models predict too low ${ }^{12} \mathrm{C} /{ }^{13} \mathrm{C}$ ratios. The situation appears even more peculiar when compared with the case of NGC 1846, for which we found C stars with higher values of $\mathrm{C} / \mathrm{O}$, but with ${ }^{12} \mathrm{C} /{ }^{13} \mathrm{C}$ between 60 and 70 , so that an AGB extra-mixing (in addition to the RGB extramixing) was invoked to reproduce the observations. The vast difference between the two clusters is illustrated in Fig. 8. In the left panel, data for NGC 1978 are compared with three models computed under different assumptions for the RGB and the AGB extra-mixing, namely no extra-mixing (black-solid line), moderate RGB extra-mixing only (green-long-dashed line), and moderate AGB extra-mixing only (blue-dashed line). All the three models have $M=1.5 M_{\odot}$ and $[\mathrm{O} / \mathrm{Fe}]=0.2$. In the right panel, data for NGC 1846 are compared with similar models. In this case, the mass is $1.8 M_{\odot}$ and the red-dashed line refers to a model with both RGB and AGB extra-mixing (see Paper I for more details on models with AGB extra-mixing). In summary, the high carbon isotopic ratio observed for the $\mathrm{C}$ stars of NGC $1978\left({ }^{12} \mathrm{C} /{ }^{13} \mathrm{C}>150\right)$ rules out both RGB and AGB extramixing, which are instead required to reproduce the evolutionary sequence of M, S, and C stars in NGC 1846.

\subsection{Alternative scenarios}

In the previous section, we did not identify a theoretical scenario that satisfactorily reproduces the whole set of stars belonging to NGC 1978 (including both the O-rich and the Crich sub-sample). Moreover, the intermediate-age LMC cluster NGC 1846, which we investigated in Paper I, presents a totally different abundance pattern, which cannot be ascribed to the same theoretical scenario. The lack of S stars is an additional peculiarity of NGC 1978. So far, our arguments have been based on the assumption that the stars in our sample possess the same initial composition, mass, and age so that they form an evolutionary sequence, in which the variations of the surface composition are due to intrinsic processes only. In this section, we speculate about possible alternative scenarios, namely:

1. the existence of two stellar populations having different ages;

2. the existence of rejuvenated stars;

3 . the activation of additional mixing at the base of the convective zones generated by a thermal pulse.

The existence of two populations proposed by Alcaíno et al. (1999) is an attractive possibility, because it might explain the 

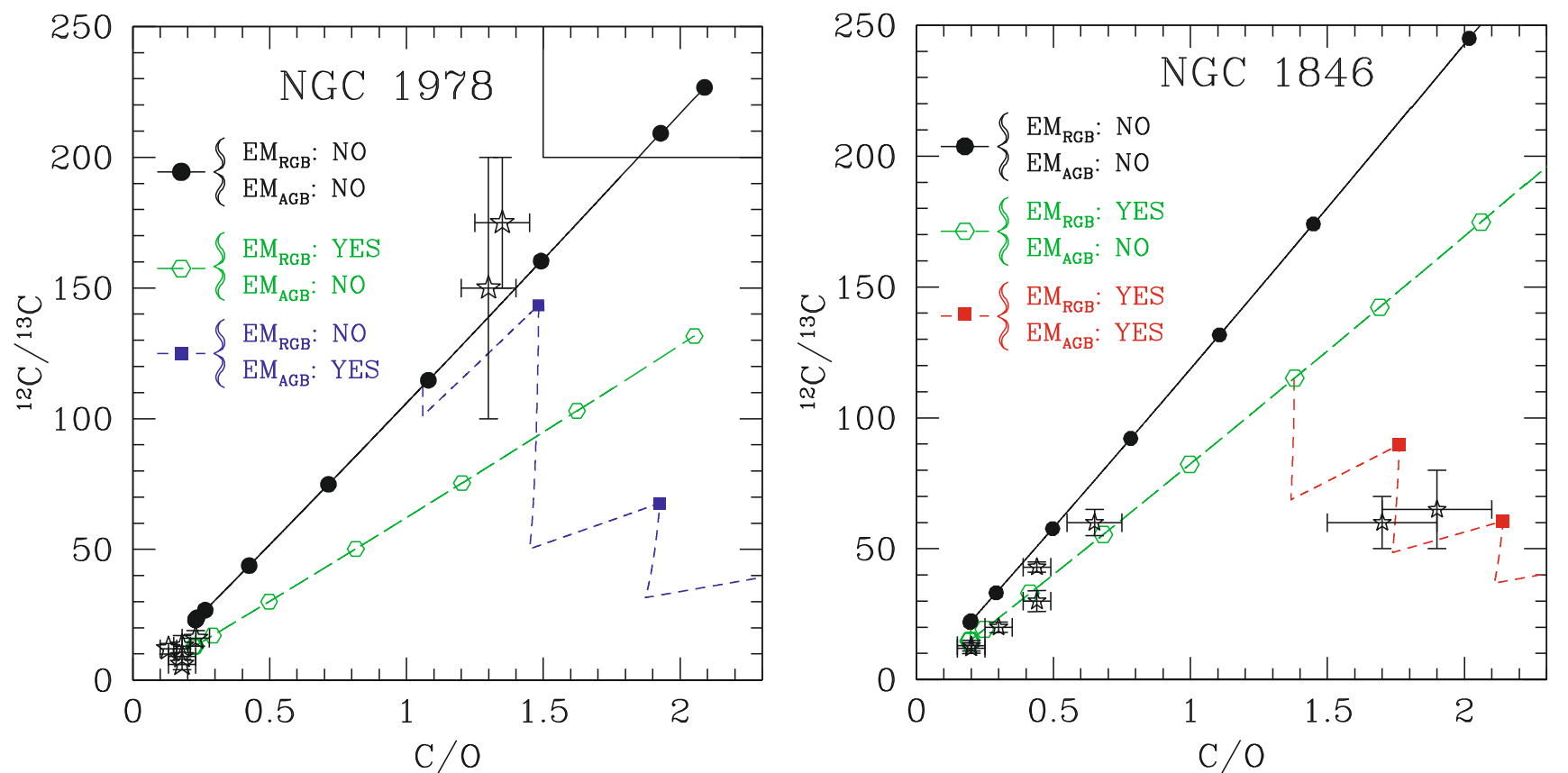

Fig. 8. Comparison between observational data and theoretical models. ${ }^{12} \mathrm{C} /{ }^{13} \mathrm{C}$ isotopic ratios vs. $\mathrm{C} / \mathrm{O}$ ratios are reported. In the left panel, we report observational data of the cluster NGC 1978, accompanied by selected theoretical tracks. As a comparison, in the right panel, we report the fitting curve for another LMC cluster (NGC 1846). The symbols along the tracks indicate the values attained during the interpulse phases (see also Fig. 7). The star symbols with error bars indicate our observations. See text for details.

dichotomy between the $\mathrm{M}$ and the $\mathrm{C}$ stars. The $\mathrm{M}$ stars would then belong to the older population. Their mass is sufficiently low for the occurrence of the RGB extra-mixing, but too low for the occurrence of the TDU during the AGB. On the contrary, the $\mathrm{C}$ stars are younger (less than $1 \mathrm{Gyr}$ ) and more massive $\left(M>2 M_{\odot}\right)$. For these stars, the persistence of the $\mu$-barrier at the base of the hydrogen-rich envelope prevented the RGB extramixing, whilst, during the AGB, TDU accounts for the observed large $C$ enhancement. However, this scenario seems unlikely. First of all, a number of other studies did not find significant metallicity, mass or age differences. Second, a cluster merger creating two populations is not considered to be a valid option (see Sect. 2). Alcaíno et al. (1999) argue that the north-western half could be more metal-rich and maybe slightly younger. The distribution of our targets in Fig. 1 does not support this idea. The $\mathrm{M}$ stars are distributed equally around the cluster centre. The carbon stars in our sample lie either in the centre or in the southeastern half of the cluster. According to Lloyd Evans (1980), there is another carbon star (LE11) located in the north-western half. Although the C stars appear to line up along the cluster's major axis, there is no indication that they concentrate in a certain region. A wide spread in metallicity would have shown up in the fitting process, yet we were able to obtain reasonable results for both oxygen-rich stars and the hottest carbon-stars with a single value for the metallicity.

The scenario of a single age stellar population does not exclude the existence of rejuvenated stars. Merging, coalescence, or mass accretion processes are often invoked to explain the Blue Stragglers observed in many GCs. Actually, a non-negligible number of stars lie above the turn-off and the sub-giant branch in the colour-magnitude diagram of NGC 1978 (Mucciarelli et al. 2007a), although we cannot exclude that those are field stars or the result of unresolved pairs. If the product of one of the above mentioned processes is a star with a mass larger than $2 M_{\odot}$, or if the rejuvenating process leaves the envelope composition enriched with heavy elements, the efficiency of the RGB extra-mixing is significantly reduced (Charbonnel \& Zahn 2007). Such a scenario might explain the high carbon isotopic ratio of the $\mathrm{C}$ stars, but the lack of $\mathrm{S}$ stars remains a mystery ${ }^{6}$. Moreover, even if we do not have a detailed statistics of the number of binary systems in NGC $1978^{7}$, it seems unlikely that two merger events occur nearly simultaneously to produce the stars B and LE6, for instance.

The last alternative scenario we have investigated regards the possible modification of the efficiency of the mixing processes taking place during the AGB phase, which is responsible for the increase of ${ }^{12} \mathrm{C}$ and ${ }^{16} \mathrm{O}$ in the envelope. A deeper TDU would increase both the carbon isotopic ratio and the $\mathrm{C} / \mathrm{O}$ ratio, thus leaving the slope of the curves in the right panel of Fig. 7 unaltered. In this scenario, the possibility of producing $\mathrm{S}$ stars as a consequence of the TDU is reduced, since the transition to the $\mathrm{C}$-star stage is more rapid. We recall that the TDU depth basically depends on the strength of the thermal pulses: stronger TPs push outward the external layers more easily, therefore inducing deeper dredge-up episodes. In turn, the strength of a thermal pulse depends on many physical and chemical properties, such as the core and the envelope mass, or the CNO content in the envelope (Straniero et al. 2003). On the other hand, an increase of the primary oxygen that is dredged up might limit the increase of the $\mathrm{C} / \mathrm{O}$ at the surface and steepen the ${ }^{12} \mathrm{C} /{ }^{13} \mathrm{C}-\mathrm{C} / \mathrm{O}$ relation. The fraction of oxygen produced by the ${ }^{12} \mathrm{C}+\alpha$ reaction increases toward the centre. Therefore, an additional mixing process, as due to a ballistic overshoot or to non-standard mechanisms (such as rotation or magnetic kick), that moves primary $\mathrm{O}$ (and $\mathrm{C}$ ) from the deep interior to the He-rich inter-shell, may indirectly explain the high carbon isotopic ratio of the carbon

\footnotetext{
${ }^{6}$ Note that our sample contains almost all AGB stars in this cluster (see Fig. 6).

7 Estimated binary fractions in other LMC and SMC clusters range from 10 (NGC 1783, Mucciarelli et al. 2007b) to $\leq 30$ per cent (Chiosi \& Vallenari 2007).
} 


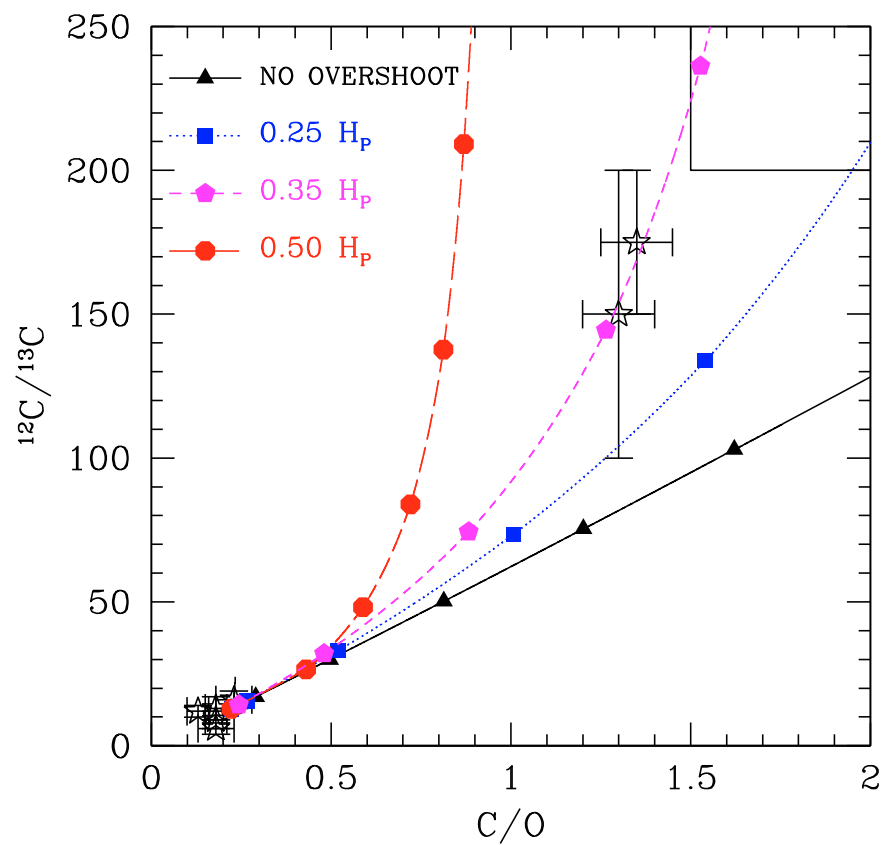

Fig. 9. ${ }^{12} \mathrm{C} /{ }^{13} \mathrm{C}$ isotopic ratio vs. $\mathrm{C} / \mathrm{O}$ ratio for a series of models including an overshoot at the base of convective zone generated by the TPs. The extension (in fraction of the pressure scale height) of the overshoot zone is indicated in the legend.

stars in NGC 1978. To shed light on this possibility, we computed some additional models, in which we applied an artificial overshoot at the base of the convective zone generated by the TPs. The initial mass adopted in the models is $1.5 M_{\odot}$, and the initial composition is oxygen-enhanced $(+0.2 \mathrm{dex})$. A moderate RGB extra-mixing $\left(T_{\mathrm{RGB}}^{\max }=2.1 \times 10^{7} \mathrm{~K}\right.$, corresponding to the case of the green long-dashed line in Fig. 7) has been included. We parametrise the additional mixing by limiting the extension of the overshoot layer to a fraction of the pressure scale height of the most internal convective mesh point. In Fig. 9, we report the resulting ${ }^{12} \mathrm{C} /{ }^{13} \mathrm{C}$ isotopic ratios as a function of $\mathrm{C} / \mathrm{O}$.

Even if these models apparently provide a simultaneous reproduction of the composition of both $\mathrm{M}$ and $\mathrm{C}$ stars, we have to stress the theoretical and observational evidence against such a process. At the He-flash peak, the inner border of the convective zone already reaches the layer where the temperature attains its maximum value. Below this point, due to the neutrino energy loss, the temperature (and the entropy) decreases, so that the deceleration due to the buoyancy is quite strong. Herwig et al. (2006) showed that even if the pressure and the entropy barrier at the base of the convective shell generated by a TP is stiff, convective plumes can penetrate into the underlying radiative layers due to $g$-mode oscillations. These structures reach down to about $2 \times 10^{8} \mathrm{~cm}$ below the formal convective border (see their Fig. 24), and develop mean vertical velocities that are about 1000 times lower than the average convective velocities. The extension of this penetration has the same order of magnitude as the overshoot zone in our models, namely $1.0 \times 10^{7} \mathrm{~cm}, 1.2 \times 10^{8} \mathrm{~cm}$, and $1.5 \times 10^{8} \mathrm{~cm}$, for $0.25 H_{\mathrm{P}}, 0.35 H_{\mathrm{P}}$, and $0.5 H_{\mathrm{P}}$, respectively (these are mean values). However, if in our 1D models the whole mass of the spherical overshoot region is efficiently mixed, the amount of mass contained in the penetrating plumes of the 3D model is certainly lower. In addition, the result of the 3D model may be affected by the assumed boundary conditions. As was stressed by Herwig et al. (2006), their models ignore $\mu$-gradients, which increase the stability and reduce mixing across the formal convective boundaries. Moreover, they found that the amplitude of the gravity waves depends both on the resolution adopted in the simulation and on the choice of the heating rate.

On the other hand, the bulk of the $\mathrm{C}$ stars in the Milky Way as well as those in NGC 1846 have ${ }^{12} \mathrm{C} /{ }^{13} \mathrm{C}$ ratios in the range from 40 to 70, and only in a few cases up to 100 (Lambert et al. 1986). This occurrence leads us to the conclusion that such an overshoot is supposedly an uncommon process. Note that the occurrence of an overshoot below the convective zone generated by a TP causes considerable changes of the physical conditions in the He-rich inter-shell. In particular, higher temperatures develop at the pulse peak and, therefore, the ${ }^{22} \mathrm{Ne}(\alpha, n)^{25} \mathrm{Mg}$ reaction rate becomes an important neutron source, even in low-mass AGB stars. In such a case, the resulting s-process nucleosynthesis would be characterised by an overproduction of neutron-rich isotopes. For instance, the expected isotopic compositions of strontium, zirconium, molybdenum, and barium would substantially differ from those measured in pre-solar SiC grains, which were produced in the cool circumstellar envelope of a past generation of C stars (see Lugaro et al. 2003). Additionally, calculated element abundances ratios such as $\mathrm{Rb} / \mathrm{Sr}$ would not match observations obtained from carbon stars (see Abia et al. 2001). For these reasons, if the overshoot from the base of the convective zone generated by TPs is non-negligible, it should be a rare event.

In summary, a clear and coherent picture of the AGB stars in the cluster NGC 1978 within the more general context of lowmass AGB stellar evolution and nucleosynthesis cannot be easily achieved. Once again, we stress the uniqueness of the $\mathrm{C}$ star sample of NGC 1978 with respect to other LMC clusters.

\section{Conclusions}

In this paper, we presented a sample of AGB stars belonging to the LMC cluster NGC 1978. Where it was possible, we derived the $\mathrm{C} / \mathrm{O}$ and the ${ }^{12} \mathrm{C} /{ }^{13} \mathrm{C}$ ratios. The spectroscopic data reveal the presence of two sub-samples, one where $\mathrm{C} / \mathrm{O}<1$ in the stellar atmosphere (oxygen-rich or M-type stars), and the other with $\mathrm{C} / \mathrm{O}>1$ (carbon-rich or C-type stars). The oxygen-rich stars present low values for the $\mathrm{C} / \mathrm{O}$ ratio (mean value 0.18 ) and the ${ }^{12} \mathrm{C} /{ }^{13} \mathrm{C}$ ratio (mean value 11 ). The spread in the $\mathrm{C} / \mathrm{O}$ ratio is restricted, all values are consistent with the mean value within the error bars. We observe a wider spread in the ${ }^{12} \mathrm{C} /{ }^{13} \mathrm{C}$ ratios. We conclude from the spectroscopic data in comparison to our evolutionary models that the TDU mechanism is not (yet) working in these stars. The carbon-rich stars of our sample instead show $\mathrm{C} / \mathrm{O}$ values and ${ }^{12} \mathrm{C} /{ }^{13} \mathrm{C}$ ratios consistent with the occurrence of TDU. A lack of $\mathrm{S}$ stars, with $\mathrm{C} / \mathrm{O}$ ratios between those of the O-rich stars and the C-rich stars, has been highlighted.

We did not find a theoretical scheme that is able to satisfactorily reproduce the chemical abundance pattern in NGC 1978. By claiming the existence of a non-standard mixing mechanism, we postulated some possible solutions. However, we are aware that too many ad hoc assumptions make our analysis objectionable, taking into account that some of them are strongly limited by observational constraints. In particular, a more consistent picture of all the additional mixing mechanisms, which are active during the RGB and the AGB phase, is required. We also discussed the scenario of multiple stellar populations and rejuvenated stars, and highlighted the differences to the abundance pattern in the cluster NGC 1846. 
Table A.1. Modified line positions of CO lines in the $K$ band.

\begin{tabular}{llllllll}
\hline \hline$\sigma_{\text {old }}\left[\mathrm{cm}^{-1}\right]$ & $\sigma_{\text {new }}\left[\mathrm{cm}^{-1}\right]$ & $E_{1}\left[\mathrm{~cm}^{-1}\right]$ & $\log g f$ & $v_{1}$ & $v_{\mathrm{u}}$ & $J_{1}$ & Isotope \\
\hline 4219.5583 & 4225.3630 & 19009.6550 & $4.865 \mathrm{E}-05$ & 1 & 3 & 95 & ${ }^{12} \mathrm{C}^{16} \mathrm{O}$ \\
4226.9718 & 4227.7190 & 14547.6172 & $6.569 \mathrm{E}-05$ & 2 & 4 & 74 & ${ }^{12} \mathrm{C}^{16} \mathrm{O}$ \\
4223.6261 & 4228.9450 & 18668.6535 & $4.782 \mathrm{E}-05$ & 1 & 3 & 94 & ${ }^{12} \mathrm{C}^{16} \mathrm{O}$ \\
4228.9365 & 4229.6010 & 14278.1804 & $6.439 \mathrm{E}-05$ & 2 & 4 & 73 & ${ }^{12} \mathrm{C}^{16} \mathrm{O}$ \\
4230.8124 & 4231.4030 & 14012.1198 & $6.310 \mathrm{E}-05$ & 2 & 4 & 72 & ${ }^{12} \mathrm{C}^{16} \mathrm{O}$ \\
4227.5739 & 4232.4430 & 18330.8030 & $4.701 \mathrm{E}-05$ & 1 & 3 & 93 & ${ }^{12} \mathrm{C}^{16} \mathrm{O}$ \\
4232.1241 & 4233.3140 & 11962.5127 & $1.167 \mathrm{E}-05$ & 0 & 2 & 81 & ${ }^{13} \mathrm{C}^{16} \mathrm{O}$ \\
4234.3009 & 4234.7650 & 13490.1716 & $6.057 \mathrm{E}-05$ & 2 & 4 & 70 & ${ }^{12} \mathrm{C}^{16} \mathrm{O}$ \\
4234.3757 & 4235.4470 & 11676.5472 & $1.144 \mathrm{E}-05$ & 0 & 2 & 80 & ${ }^{13} \mathrm{C}^{16} \mathrm{O}$ \\
4235.9151 & 4236.3250 & 13234.3055 & $5.932 \mathrm{E}-05$ & 2 & 4 & 69 & ${ }^{12} \mathrm{C}^{16} \mathrm{O}$ \\
4237.4434 & 4237.8040 & 12981.8588 & $5.809 \mathrm{E}-05$ & 2 & 4 & 68 & ${ }^{12} \mathrm{C}^{16} \mathrm{O}$ \\
4238.8865 & 4239.2040 & 12732.8418 & $5.687 \mathrm{E}-05$ & 2 & 4 & 67 & ${ }^{12} \mathrm{C}^{16} \mathrm{O}$ \\
4240.2450 & 4240.5230 & 12487.2648 & $5.566 \mathrm{E}-05$ & 2 & 4 & 66 & ${ }^{12} \mathrm{C}^{16} \mathrm{O}$ \\
4241.5194 & 4241.7630 & 12245.1376 & $5.447 \mathrm{E}-05$ & 2 & 4 & 65 & ${ }^{12} \mathrm{C}^{16} \mathrm{O}$ \\
\hline
\end{tabular}

Narrower constraints for stellar evolutionary models could be derived from atmospheric models including a set of accurate molecular line data, especially in the case of carbon stars. Additionally, the more evolved a star is (thus having a higher luminosity and $\mathrm{C} / \mathrm{O}$ ratio), the more dynamic effects influence the spectral appearance. From the observational point of view, improvements could be achieved by means of the choice of the observed wavelength regions, some of which are less crowded with molecular lines. Moreover a comparison between low-resolution data and high-resolution data should be explored further (cf. Wahlin et al. 2006). If low-resolution data deliver robust $\mathrm{C} / \mathrm{O}$ ratios (and stellar parameters), the isotopic ratios could be derived in the high-resolution spectra more accurately.

In conclusion, we affirm that there is much room for improvement. As a first step, we are currently acquiring new data to increase our statistics by studying more AGB stars in other LMC globular clusters. In this way, we could shed light on the puzzling abundance pattern that we found in NGC 1978.

Acknowledgements. MTL and TL acknowledge funding by the Austrian Science Fund FWF (projects P-18171 and P-20046). MTL has been supported by the Austrian Academy of Sciences (DOC programme). OS and SC have been supported by the MIUR Italian Grant Program PRIN 2006. BA acknowledges funding by the Austrian Science Fund FWF (project P-19503). BA acknowledges funding by the contract ASI-INAF I/016/07/0. Based on observations obtained at the Gemini Observatory, which is operated by the Association of Universities for Research in Astronomy, Inc., under a cooperative agreement with the NSF on behalf of the Gemini partnership: the National Science Foundation (United States), the Science and Technology Facilities Council (United Kingdom), the National Research Council (Canada), CONICYT (Chile), the Australian Research Council (Australia), Ministério da Ciência e Tecnologia (Brazil) and Ministerio de Ciencia, Tecnología e Innovación Productiva (Argentina). The observations were obtained with the Phoenix infrared spectrograph, which was developed and is operated by the National Optical Astronomy Observatory. The spectra were obtained as part of the programs GS-2006B-C-6 and GS-2008AQ-65. This publication makes use of data products from the Two Micron All Sky Survey, which is a joint project of the University of Massachusetts and the Infrared Processing and Analysis Center/California Institute of Technology, funded by the National Aeronautics and Space Administration and the National Science Foundation.

\section{Appendix A: Changes to the $\mathrm{CO}$ line list}

To match the positions of the CO lines in the recorded $K$-band spectra, we had to modify some transition frequencies as given in the list of Goorvitch $(1994)^{8}$. The wavenumbers were taken from the Infrared Atlas of the Arcturus Spectrum (Hinkle et al. 1995).

\footnotetext{
8 Available at the CDS: http://vizier.cfa.harvard.edu/ viz-bin/VizieR?-source=J/ApJS/95/535.
}

The changes are documented in Table A.1. The given quantities are the old and new transition frequency $(\sigma)$, the lower state term energy $E_{1}$, the logarithm of the $g f$-value $(\log g f)$, the lower and upper state vibrational quantum numbers $\left(v_{1}\right.$ and $\left.v_{\mathrm{u}}\right)$, the lower state angular momentum number $\left(J_{1}\right)$, and the isotope identification.

At a later point in the analysis, we compared the corrections of the line positions to the $\mathrm{CO}$ data given by Chandra et al. (1996). The wavenumbers from the Arcturus atlas are in good agreement with their transition frequencies. The differences are less than $0.04 \mathrm{~cm}^{-1}$ for the 1-3 vibration transitions and less than $0.01 \mathrm{~cm}^{-1}$ for the other lines. However, we did not use the Chandra et al. (1996) data, because the line strengths of the ${ }^{12} \mathrm{CO}$ 3-0 band head in the $H$ band from this list are severely underestimated and therefore unusable for the analysis.

\section{References}

Abia, C., Busso, M., Gallino, R., et al. 2001, ApJ, 559, 1117

Alcaíno, G., Liller, W., Alvarado, F., et al. 1999, A\&AS, 135, 103

Anders, E., \& Grevesse, N. 1989, Geochim. Cosmochim. Acta, 53, 197 Aringer, B. 2000, Ph.D. Thesis, University of Vienna

Aringer, B., Girardi, L., Nowotny, W., Marigo, P., \& Lederer, M. T. 2009, A\&A in press, [ArXiv: 0905.4415]

Aringer, B., Kerschbaum, F., \& Jørgensen, U. G. 2002, A\&A, 395, 915

Bessell, M. S., Wood, P. R., \& Evans, T. L. 1983, MNRAS, 202, 59

Bonnarel, F., Fernique, P., Bienaymé, O., et al. 2000, A\&AS, 143, 33

Busso, M., Gallino, R., \& Wasserburg, G. J. 1999, ARA\&A, 37, 239

Carpenter, J. M. 2001, AJ, 121, 2851

Carrera, R., Gallart, C., Hardy, E., Aparicio, A., \& Zinn, R. 2008, AJ, 135, 836

Castellani, V., Degl'Innocenti, S., Marconi, M., Prada Moroni, P. G., \& Sestito, P. 2003, A\&A, 404, 645

Chandra, S., Maheshwari, V. U., \& Sharma, A. K. 1996, A\&AS, 117, 557

Charbonnel, C., \& Zahn, J.-P. 2007, A\&A, 467, L15

Chieffi, A., Limongi, M., \& Straniero, O. 1998, ApJ, 502, 737

Chiosi, E., \& Vallenari, A. 2007, A\&A, 466, 165

Cioni, M.-R. L., van der Marel, R. P., Loup, C., \& Habing, H. J. 2000, A\&A, 359,601

Cole, A. A., Tolstoy, E., Gallagher, III, J. S., \& Smecker-Hane, T. A. 2005, AJ, 129,1465

Cristallo, S., Straniero, O., Lederer, M. T., \& Aringer, B. 2007, ApJ, 667, 489

Cristallo, S., Straniero, O., Gallino, R., et al. 2009, ApJ, in press, [ArXiv: 0902.0243]

Davis, S., Wallace, L., Brault, J., \& Engleman, R. 2005, The CN spectrum from the infrared to the ultraviolet (NSO Technical Report)

de Laverny, P., Abia, C., Domínguez, I., et al. 2006, A\&A, 446, 1107

Denissenkov, P. A., \& Pinsonneault, M. 2008, ApJ, 684, 626

Eggleton, P. P., Dearborn, D. S. P., \& Lattanzio, J. C. 2008, ApJ, 677, 581

Ferraro, F. R., Mucciarelli, A., Carretta, E., \& Origlia, L. 2006, ApJ, 645, L33

Fischer, P., Welch, D. L., \& Mateo, M. 1992, AJ, 104, 1086

Frogel, J. A., Mould, J., \& Blanco, V. M. 1990, ApJ, 352, 96 
Gautschy-Loidl, R., Höfner, S., Jørgensen, U. G., \& Hron, J. 2004, A\&A, 422, 289

Girardi, L., Chiosi, C., Bertelli, G., \& Bressan, A. 1995, A\&A, 298, 87

Goorvitch, D. 1994, ApJS, 95, 535

Goorvitch, D., \& Chackerian, Jr., C. 1994, ApJS, 91, 483

Goudfrooij, P., Puzia, T. H., Kozhurina-Platais, V., \& Chandar, R. 2009, AJ, 137, 4988

Gratton, R. G., Carretta, E., Matteucci, F., \& Sneden, C. 2000, A\&A, 358, 671

Grevesse, N., \& Noels, A. 1993, Phys. Scri. Vol. T, 47, 133

Grocholski, A. J., Sarajedini, A., Olsen, K. A. G., Tiede, G. P., \& Mancone, C. L. 2007, AJ, 134, 680

Gustafsson, B., Bell, R. A., Eriksson, K., \& Nordlund, A. 1975, A\&A, 42, 407

Gustafsson, B., Edvardsson, B., Eriksson, K., et al. 2008, A\&A, 486, 951

Harris, M. J., Lambert, D. L., Hinkle, K. H., Gustafsson, B., \& Eriksson, K. 1987, ApJ, 316, 294

Herwig, F. 2000, A\&A, 360, 952

Herwig, F., Blöcker, T., \& Driebe, T. 2000, Mem. Soc. Astron. Ital., 71, 745

Herwig, F., Freytag, B., Hueckstaedt, R. M., \& Timmes, F. X. 2006, ApJ, 642, 1057

Hill, V., François, P., Spite, M., Primas, F., \& Spite, F. 2000, A\&A, 364, L19

Hinkle, K., Wallace, L., \& Livingston, W. C. 1995, Infrared atlas of the Arcturus spectrum, 0.9-5.3 microns (San Francisco, Calif.: Astronomical Society of the Pacific)

Hinkle, K. H., Hall, D. N. B., \& Ridgway, S. T. 1982, ApJ, 252, 697

Hinkle, K. H., Cuberly, R. W., Gaughan, N. A., et al. 1998, in Infrared Astronomical Instrumentation, Presented at the Society of Photo-Optical Instrumentation Engineers (SPIE) Conference, ed. A. M. Fowler, Proc. SPIE, 3354,810

Hinkle, K. H., Blum, R. D., Joyce, R. R., et al. 2003, in 4834, Society of Photo-Optical Instrumentation Engineers (SPIE) Conference Series, ed. P. Guhathakurta, 353

Houdashelt, M. L., Bell, R. A., Sweigart, A. V., \& Wing, R. F. 2000, AJ, 119, 1424

Iben, Jr., I., \& Renzini, A. 1983, ARA\&A, 21, 271

Johnson, H. B. 1994, in Lecture Notes in Physics, Molecules in the Stellar Environment (Berlin: Springer Verlag), ed. U. G. Jorgensen, IAU Coll., 146, 234

Jørgensen, U. G. 1997, ed. E. F. van Dishoeck, IAU Symp., 178, 441

Jørgensen, U. G., Johnson, H. R., \& Nordlund, A. 1992, A\&A, 261, 263

Karakas, A., \& Lattanzio, J. C. 2007, PASA, 24, 103

Kupka, F. G., Ryabchikova, T. A., Piskunov, N. E., Stempels, H. C., \& Weiss, W. W. 2000, BaltA, 9, 590
Lambert, D. L., Gustafsson, B., Eriksson, K., \& Hinkle, K. H. 1986, ApJS, 62, 373

Lebzelter, T., Lederer, M. T., Cristallo, S., et al. 2008, A\&A, 486, 511

Lederer, M. T., \& Aringer, B. 2009, A\&A, 494, 403

Lloyd Evans, T. 1980, MNRAS, 193, 87

Lloyd Evans, T. 1983, MNRAS, 204, 985

Loidl, R., Lançon, A., \& Jørgensen, U. G. 2001, A\&A, 371, 1065

Lugaro, M., Davis, A. M., Gallino, R., et al. 2003, ApJ, 593, 486

Mackey, A. D., \& Broby Nielsen, P. 2007, MNRAS, 379, 151

Matsuura, M., Zijlstra, A. A., van Loon, J. T., et al. 2005, A\&A, 434, 691

McSaveney, J. A., Wood, P. R., Scholz, M., Lattanzio, J. C., \& Hinkle, K. H. 2007, MNRAS, 378, 1089

Mucciarelli, A., Carretta, E., Origlia, L., \& Ferraro, F. R. 2008, AJ, 136, 375

Mucciarelli, A., Ferraro, F. R., Origlia, L., \& Fusi Pecci, F. 2007a, AJ, 133, 2053

Mucciarelli, A., Origlia, L., \& Ferraro, F. R. 2007b, AJ, 134, 1813

Nollett, K. M., Busso, M., \& Wasserburg, G. J. 2003, ApJ, 582, 1036

Olszewski, E. W., Schommer, R. A., Suntzeff, N. B., \& Harris, H. C. 1991, AJ, 101,515

Piotto, G., Bedin, L. R., Anderson, J., et al. 2007, ApJ, 661, L53

Querci, F., Querci, M., \& Tsuji, T. 1974, A\&A, 31, 265

Renzini, A. 2008, MNRAS, 391, 354

Reyniers, M., Abia, C., van Winckel, H., et al. 2007, A\&A, 461, 641

Schommer, R. A., Suntzeff, N. B., Olszewski, E. W., \& Harris, H. C. 1992, AJ, 103,447

Skrutskie, M. F., Cutri, R. M., Stiening, R., et al. 2006, AJ, 131, 1163

Smith, V. V., \& Lambert, D. L. 1990, ApJS, 72, 387

Smith, V. V., Hinkle, K. H., Cunha, K., et al. 2002, AJ, 124, 3241

Stancliffe, R. J., Tout, C. A., \& Pols, O. R. 2004, MNRAS, 352, 984

Stanghellini, L., Shaw, R. A., \& Gilmore, D. 2005, ApJ, 622, 294

Straniero, O., Chieffi, A., Limongi, M., et al. 1997, ApJ, 478, 332

Straniero, O., Domínguez, I., Cristallo, R., \& Gallino, R. 2003, PASA, 20, 389

Straniero, O., Gallino, R., \& Cristallo, S. 2006, Nucl. Phys. A, 777, 311

van den Bergh, S. 2008, AJ, 135, 1731

Wahlin, R., Eriksson, K., Gustafsson, B., et al. 2006, Mem. Soc. Astron. It., 77, 955

Wasserburg, G. J., Boothroyd, A. I., \& Sackmann, I.-J. 1995, ApJ, 447, L37

Wilson, R. E. 1953, General catalogue of stellar radial velocities (Washington: Carnegie Institution of Washington)

Wood, P. R., Bessell, M. S., \& Fox, M. W. 1983, ApJ, 272, 99

Yong, D., Meléndez, J., Cunha, K., et al. 2008, ApJ, 689, 1020 